\title{
A spin foam model without bubble divergences
}

\author{
Alejandro Perez and Carlo Rovelli \\ Centre de Physique Théorique - CNRS, Case 907, Luminy, F-13288 Marseille, France, and \\ Physics Department, University of Pittsburgh, Pittsburgh, Pa 15260, USA
}

\begin{abstract}
We present a spin foam model in which the fundamental "bubble amplitudes" (the analog of the one-loop corrections in quantum field theory) are finite as the cutoff is removed. The model is a natural variant of the field theoretical formulation of the Barrett-Crane model. As the last, the model is a quantum BF theory plus an implementation of the constraint that reduces BF theory to general relativity. We prove that the fundamental bubble amplitudes are finite by constructing an upper bound, using certain inequalities satisfied by the Wigner $(3 n) j$-symbols, which we derive in the paper. Finally, we present arguments in support of the conjecture that the bubble diagrams of the model are finite at all orders.
\end{abstract}

\section{INTRODUCTION}

To unlock the puzzle of quantum gravity, and understand quantum spacetime, we have to learn how to treat general relativistic quantum field theories in a background independent fashion [1]. A surprising number of research directions aimed at exploring background independent quantum field theory have recently been converging towards the spin foam formalism [2]

A spin foam model can be seen as a rigorous implementation of the Wheeler-Misner-Hawking [8,9] sum over geometries formulation of quantum gravity. The foam-like geometries summed over are spin foams, or colored 2complexes. A 2-complex $J$ is a (combinatorial) set of elements called "vertices" $v$, "edges" $e$ and "faces" $f$, and a boundary relation among these, such that an edge is bounded by two vertices, and a face is bounded by a cyclic sequence of contiguous edges (edges sharing a vertex). A spin foam is a 2-complex plus a "coloring" $N$, that is an assignment of an irreducible representation $N_{f}$ of a given group $G$ to each face $f$ and of an intertwiner $i_{e}$ to each edge $e$. The model is defined by the partition function:

$$
Z=\sum_{J} \mathcal{N}(J) \sum_{N} \prod_{f \in J} \Delta_{N_{f}} \prod_{v \in J} A_{v}(N),
$$

where $\Delta_{N}$ is the dimension of the representation $N, A_{v}(N)$ is an amplitude associated to vertices: a given function of the colorings of the faces and edges adjacent to the vertex; and $\mathcal{N}(J)$ is a normalization factor for each 2-complex.

Let us mention a few of the research directions that, following very different paths, have converged to models of this kind. One of the oldest models of this type is the Ponzano and Regge formulation of $2+1$ gravity [10. The 2complexes in this case are the 2-skeleta of the dual of Regge triangulations, and the vertex amplitude turns out to be simply a Wigner $S U(2) 6 \mathrm{j}$ symbol. In loop quantum gravity [11], spin foams emerge as histories (in coordinate time) of quantum states of the geometry [5], that is, histories of spin networks [12,13]. In this case, the vertex amplitude $A_{v}(c)$ is given by the matrix elements of the Hamiltonian constraint. In covariant lattice approaches, the sum over colors corresponds to the integration over group elements associated to links, expressed in a ("Fourier") mode expansion over the group. In this case the vertex amplitude $A_{v}(c)$ is a discretized version of (the exponential of) the Lagrangian density [14,15]. In topological field theories, the vertex function is a natural object in the representation theory of the group $G$, satisfying a set of identities that assure triangulation independence 17 20. Finally, in the modifications of topological quantum field theories related to quantum general relativity [7],14,16], the topological field theory vertex amplitude is altered in order to incorporate a quantum version of the constraints that reduces the BF topological field theory 21,22] to general relativity.

Spin foams are very much analogous to Feynman diagrams. In references [23,24, indeed, it is shown that the sum (11) can be obtained as a Feynman expansion of a field theory over a group manifold, whose interaction terms determine the vertex amplitude (see also [25]). In this context, spacetime emerges from a Feynman expansion, as in the old $2 \mathrm{~d}$ quantum gravity matrix models, or zero-dimensional string theory [26].

As in the Feynman expansions of a conventional field theory, one expects divergences to appear in the sum. There are two types of potential divergences: the ones associated to the sum over the colors of a fixed 2-complex, and the ones associated with the sum over 2-complexes. Here we consider only divergences of the first kind. In conventional Feynman diagrammatics, divergences are originated by integrating over the momenta circulating along closed loops, because momentum conservation at the vertices relates the momenta of adjacent propagators. In a spin foams model, the sum is over representations associated to faces, and a constraint analogous to momentum conservation is provided 
by the requirement of the existence of non trivial intertwiners on the edges. Consequently, divergences are associated not to loops, as in quantum field theory, but rather to bubbles: collections of faces forming a closed surface [27]. A way to control bubble divergences is to replace the group $G$ with a $q$-deformed group, choosing $q$ such that $q^{n}=1$, and to sum only over the finite set of proper representations of the quantum group. This is done, for instance, in the Turaev-Viro [28] finite version of the Ponzano-Regge theory. The parameter $q$ plays the role of a cutoff, and the physical theory is recovered by appropriately taking the $q \rightarrow 1$ limit.

The significance of the bubble divergences depends on whether the model is topological. A topological field theory is a diffeomorphism invariant theory which does not have local degrees of freedom, but only global ones. General relativity in 3 spacetime dimensions and BF theory are topological theories. On the other hand, a nontopological diffeomorphism-invariant theory is a theory, such as general relativity in $4 \mathrm{~d}$, which is generally covariant but, nevertheless, has local degrees of freedom (waves). In the context of spin foam models, the fact that a theory is topological is reflected in the fact that the amplitude of a fixed 2-complex in (11) is independent from the 2-complex (triangulation independence ones the manifold, i.e., the topology is fixed). More precisely, in general, in a topological model, bubble amplitudes diverges in the $q \rightarrow 1$ limit, but topological invariance implies that the sum over colorings is the same as the one of a 2-complex in which the bubble has been removed, up to a divergent overall factor that depends only on the cutoff. In this case, therefore, divergent diagrams do not provide any additional information. The fact that a topological theory does not have local degrees of freedom is reflected in the triviality of all its "radiative corrections" (bubble diagrams). The consequence is that the sum over 2-complexes is trivial, and can be dropped, thus dropping all 2-complexes with bubbles.

The situation is different in the non-topological context, and in particular, for quantum general relativity. In this case, the "radiative corrections", that is, the bubble amplitudes, are the ones that carry the information about the quantum behavior of the local degrees of freedom of the theory. In this context the sum over 2-complexes is necessary in order to capture all the degrees of freedom of the theory, and bubble amplitudes are physically important.

Examples of non-topological models are the covariant expansion in coordinate time obtained from loop quantum gravity, the Iwasaki [16] and the Reisenberger [21] models, and the Barrett-Crane model. The Barrett-Crane model is a non-topological modification of a topological model: $4 \mathrm{~d} S O(4) \mathrm{BF}$ theory, or the TOCY (Turaev-OoguriBarrett-Yetter) model 17 20]. It is well known that $4 \mathrm{~d} S O(4) \mathrm{BF}$ theory has a peculiar relation with 4d Euclidean general relativity: general relativity can be seen as a $4 \mathrm{~d} S O(4) \mathrm{BF}$ theory plus a constraint, which has an intriguing geometrical interpretation. The Barrett-Crane model is a modification of the TOCY model in which this constraint is implemented in the quantum theory [29, 30 ]. As a consequence of the constraints, topological invariance is lost and the model acquires local degrees of freedom. Radiative corrections associated to bubble diagrams carry non trivial physical information, but, unfortunately, diverge [27]. In order to be able to extract physical information from this model it is necessary to deal with these divergences.

In a companion paper [27, we have begun a general study of the bubble amplitudes and the possible ways of renormalizing away their infinities (see also 34]). In particular, we have studied the version of the Barrett-Crane model developed in [23], in which the sum over complexes is explicitly implemented by obtaining the spin foam model from a field theory over a group manifold. In the course of this analysis, we have stumbled across a remarkable simple modification of the action of the Barrett-Crane model which leads to finite fundamental bubble amplitudes. 1 We present this model in the present paper. We also argue that all "radiative corrections" in the model are likely to be finite. The model we present shares with the original Barrett-Crane model the feature that makes it an intriguing candidate for a quantum theory of (Euclidean) $4 \mathrm{~d}$ general relativity: that is, it is another implementation of the constraint that reduces BF to GR. As a result, we obtain a theory, formally related to Euclidean quantum general relativity, presumably finite at all orders in the expansion over 2-complexes.

The paper is organized as follows. In section II, we recall the field theory formulation of spin foam models by discussing the TOCY model [17 20], and the Barrett-Crane model; then we introduce the new model. In section III, we study the model in configuration space. We define and compute the amplitudes corresponding to the fundamental bubble diagrams. We prove finiteness of the 1-bubble diagram. In section IV, we study the model in momentum space where we show that the interaction vertex of the theory is the Barrett-Crane vertex. We also give another proof of the finiteness of the 5-bubble amplitude. In the appendix we review some known facts about the representation theory of $S O(4)$, and we prove certain inequalities satisfied by the Barrett-Crane vertex amplitude, the $6 j$, and the $15 j$-symbols. We use these inequalities to show the finiteness of the fundamental bubble diagrams. They generalize to the $3 N j$-symbols.

\footnotetext{
${ }^{1}$ The precise meaning of the fundamental bubble diagrams is explained below. They represent the simplest potentially divergent graphs in analogy to the one loop corrections in standard QFT.
} 


\section{DEFINITION OF THE MODEL}

We begin by recalling the formulation of quantum BF theory, and of the Barrett-Crane Euclidean quantum gravity, as field theories on a group manifold. These were first developed by Ooguri in [18] and by DePietri et al in [23]. Then we introduce the new model.

\section{A. TOCY model as a QFT over a group manifold}

A spin foam model can be cast in the form of a field theory over a group manifold [23,24. The simplest of these theories is given by the TOCY [17 20 topological model, corresponding to the quantization of $S O(4) 4$-dimensional BF theory. We begin by describing this model, which allows us to introduce definitions relevant in the sequel. The model is defined by the action.

$$
\begin{aligned}
S[\phi]= & \frac{1}{2} \int d g_{1} \ldots d g_{4} \phi^{2}\left(g_{1}, g_{2}, g_{3}, g_{4}\right)+\frac{\lambda}{5 !} \int d g_{1} \ldots d g_{10} \phi\left(g_{1}, g_{2}, g_{3}, g_{4}\right) \\
& \phi\left(g_{4}, g_{5}, g_{6}, g_{7}\right) \phi\left(g_{7}, g_{3}, g_{8}, g_{9}\right) \phi\left(g_{9}, g_{6}, g_{2}, g_{10}\right) \phi\left(g_{10}, g_{8}, g_{5}, g_{1}\right) .
\end{aligned}
$$

Here $g_{i} \in S O(4)$ and the field $\phi$ is a function over $S O(4)^{4}$. All the integrals in this paper are in the normalized Haar measure. The field $\phi$ is required to be invariant under any permutation of its arguments; that is, $\phi\left(g_{1}, g_{2}, g_{3}, g_{4}\right)=$ $\phi\left(g_{\sigma(1)}, g_{\sigma(2)}, g_{\sigma(3)}, g_{\sigma(4)}\right)$, where $\sigma$ is any permutation of four elements; and under the simultaneous right action of $S O(4)$ on its four arguments:

$$
\phi\left(g_{1}, g_{2}, g_{3}, g_{4}\right)=\phi\left(g_{1} g, g_{2} g, g_{3} g, g_{4} g\right) \quad \forall g \in S O(4)
$$

Let us introduce some simplification in the notation. We write $\phi\left(g_{1}, g_{2}, g_{3}, g_{4}\right)$ as $\phi\left(g_{i}\right)$, and we write the (2) as

$$
S[\phi]=\int d g_{i}\left[\phi\left(g_{i}\right)\right]^{2}+\frac{\lambda}{5 !} \int d g_{i}\left[\phi\left(g_{i}\right)\right]^{5},
$$

where, notice, the fifth power has to be interpreted as in (2).

Instead of requiring that the field satisfies property (3), we can also define the theory in terms of a generic field $\phi$ and project it on the space of the fields satisfying (3) by integrating over the group. That is, we can define the theory by the action

$$
S[\phi]=\int d g_{i}\left[P_{g} \phi\left(g_{i}\right)\right]^{2}+\frac{\lambda}{5 !} \int d g_{i}\left[P_{g} \phi\left(g_{i}\right)\right]^{5}
$$

where the field is now arbitrary (except for the permutation symmetry), and the operator $P_{g}$ is defined by

$$
P_{g} \phi\left(g_{i}\right) \equiv \int d \gamma \phi\left(g_{i} \gamma\right)
$$

where $\gamma \in S O(4)$.

Let us write the Feynman rules of the theory in coordinate space. To this aim, we write the action as

$$
S[\phi]=\frac{1}{2} \int d g_{i} d \tilde{g}_{i} \phi\left(g_{i}\right) \mathcal{K}\left(g_{i}, \tilde{g}_{i}\right) \phi\left(\tilde{g}_{i}\right)+\frac{\lambda}{5 !} \int d g_{i j} \mathcal{V}\left(g_{i j}\right) \phi\left(g_{1 j}\right) \phi\left(g_{2 j}\right) \phi\left(g_{3 j}\right) \phi\left(g_{4 j}\right) \phi\left(g_{5 j}\right)
$$

in the last integral, $i \neq j$. Clearly, $\phi\left(g_{1 j}\right)=\phi\left(g_{12}, g_{13}, g_{14}, g_{15}\right)$, and so on.

The kinetic operator $\mathcal{K}\left(g_{i}, \tilde{g}_{i}\right)$ can be written as

$$
\mathcal{K}\left(g_{i}, \tilde{g}_{i}\right)=\sum_{\sigma} \int d \gamma \prod_{i=1}^{4} \delta\left(g_{i} \gamma \tilde{g}_{\sigma(i)}^{-1}\right) .
$$

The propagator is the inverse of the kinetic operator, in the space of the gauge invariant fields. The operator $\mathcal{K}$ is a projector (i.e., $\mathcal{K}^{2}=\mathcal{K}$ ): its inverse in the subspace of gauge invariant fields corresponds to itself. The propagator of the theory is then simply 


$$
\mathcal{P}\left(g_{i}, \tilde{g}_{i}\right)=\mathcal{K}\left(g_{i}, \tilde{g}_{i}\right)
$$

The propagator is formed by 4 delta functions (plus the symmetrization and the integration over the group). Its structure can therefore be represented graphically as in Fig. 1, in which every line represents one of the delta functions in (9).

FIG. 1. The structure of the propagator.

The potential term can be written as

$$
\mathcal{V}\left(g_{i j}\right)=\frac{1}{5 !} \int d \beta_{i} \prod_{i<j} \delta\left(g_{i j} \beta_{i}^{-1} \beta_{j} g_{j i}^{-1}\right)
$$

where $\beta_{i} \in S O(4)$. By introducing the notation $\rho_{j i}=g_{j i}^{-1} g_{i j}$, which satisfies the property $\rho_{i j}^{-1}=\rho_{j i}$, and rearranging the arguments of the delta functions using the fact that $\delta\left(g_{1} g_{2}\right)=\delta\left(g_{2} g_{1}\right)$, we can write the potential term as a function of ten variables only

$$
\mathcal{V}\left(\rho_{i j}\right)=\frac{1}{5 !} \int d \beta_{i} \prod_{i<j} \delta\left(\beta_{j} \rho_{j i} \beta_{i}^{-1}\right) .
$$

The structure of the vertex is represented in Fig. 2, where each line represents one of the delta functions that appear in the previous expression.

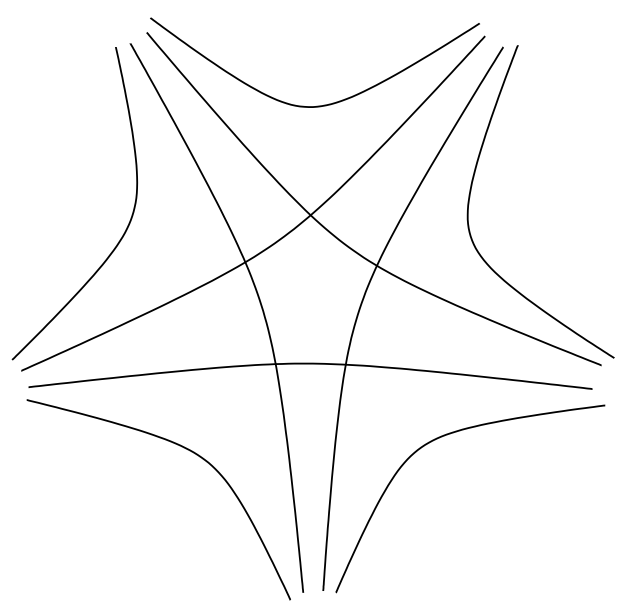

FIG. 2. The structure of the interaction vertex.

From now on we will use the Greek letter $\beta$ to denote the symmetrization integration variables associated to vertices, while we reserve the letter $\gamma$ for propagators.

The Feynman diagrams of the theory are obtained by connecting the five-valent vertices with propagators. At the open ends of propagators and vertices there are the four group variables corresponding to the arguments of the field. For a fixed permutation $\sigma$ in each propagator, one can follow the sequence of delta functions with common arguments across vertices and propagators. On a closed graph, each such sequence must close. By associating a surface to each such sequence of propagators, we construct a 2-complex [23]. Thus, by expanding in Feynman diagrams and in the sum over permutations in (8), we obtain a sum over 2-complexes. In other words, a 2-complex is given by a certain 
vertices-propagators topology plus a fixed choice of a permutation on each propagator. From now on, a "Feynman diagram", or simply a diagram, will denote one such 2-complexes.

According to the standard Feynman rules deduced from the form of the action, the amplitude of any diagram will be given as an integral over the internal coordinates -in this case $S O(4)$ group elements- of the product of the corresponding propagators and vertices. Thus, the amplitude of any Feynman diagram is given by an integral of a product of delta functions over a (compact) group manifold. From (9) and (10) we observe that the integrand defining this amplitude correspond to a product of delta functions related to each other according to Fig. 11 and 2.

Working in configuration space, one can easily prove the topological invariance of the theory using the Feynman rules derived from (5) 27, 18]. If one expands the action (5) in terms of irreducible representations using the PeterWeyl theorem, then the amplitude of a Feynman diagram $J$ is

$$
A(J)=\sum_{N} \prod_{f} \Delta_{N_{f}} \prod_{v} 15 j\left(N_{v}\right)
$$

where $15 j\left(N_{v}\right)$ denotes the $15 j$-symbol constructed out of the 10 colors of the surfaces meeting at the vertex plus the five colors of the intertwiners corresponding to the five edges defining the vertex. The sum goes over all possible compatible colorings of faces and intertwiners in the 2-complex.

\section{B. The Barrett-Crane model in coordinate space}

Now we describe the Barrett-Crane model as a field theory over $S O(4)^{4}$. Consider the fundamental representation of $S O(4)$, defined on $\Re^{4}$, and pick a fixed direction $\hat{t}$ in $\Re^{4}$. Let $H$ be the $S O(3)$ subgroup of $S O(4)$ that leaves $\hat{t}$ invariant. We define the projector $P_{h}$

$$
P_{h} \phi\left(g_{i}\right) \equiv \int d h_{i} \phi\left(g_{i} h_{i}\right)
$$

where $h_{i} \in H$. From now on, the letter $h$ will always indicate an element in $H=S O(3)$, while we use $g, \beta, \gamma$, and $\rho$ for elements in $G=S O(4)$. The Barrett-Crane model is given by the action:

$$
S[\phi]=\int d g_{i}\left[P_{g} P_{h} \phi\left(g_{i}\right)\right]^{2}+\frac{\lambda}{5 !} \int d g_{i}\left[P_{g} P_{h} \phi\left(g_{i}\right)\right]^{5},
$$

where the notation is as in the previous section and the fields are assumed to be symmetric under permutations of their four arguments. In [23], it is shown that the Feynman expansion of (14) gives the Barrett-Crane spin foam model, summed over 2-complexes.

Notice that the action (14) is simply obtained by adding the $P_{h}$ projection to the the action (5) of the topological TOCY. The projector $P_{h}$ projects the field over the linear subspace of the fields that are constants on the orbits of $H$ in $G$, that is, that satisfy

$$
\phi\left(g_{1}, g_{2}, g_{3}, g_{4}\right)=\phi\left(g_{1} h_{1}, g_{2} h_{2}, g_{3} h_{3}, g_{4} h_{4}\right) \quad \forall h_{i} \in H .
$$

When expanding the field in modes, that is, on the irreducible representations of $S O(4)$, this projection is equivalent to restricting the expansion to the representations in which there is a vector invariant under the subgroup $H$ (because the projection projects on such invariant vectors). The representations in which such invariant vectors exist are precisely the "simple" representations, namely the ones in which the spin of the self dual sector is equal to the spin of the antiselfdual sector. In turn, the simple representations are the ones whose generators have equal selfdual and antiself dual components, and this equality, under identification of the $S O(4)$ generator with the $B$ field of $B F$ theory is precisely the constraint that reduces $B F$ theory to GR. Alternatively, this constraint allows one to identify the generators as bivectors defining elementary surfaces in $4 \mathrm{~d}$, and thus to interpret the coloring of a two-simplex as the choice of a (discretized) $4 \mathrm{~d}$ geometry [2, 2, 30].

Using equations (6), and (13) it is straightforward to compute the kinetic operator and the interaction vertex of the Barrett-Crane model in coordinate space. The kinetic operator of the theory is

$$
\mathcal{K}\left(g_{i}, \tilde{g}_{i}\right)=\int d \gamma d h_{i} \prod_{i} \delta\left(g_{i} h_{i} \gamma \tilde{h}_{i} \tilde{g}_{i}^{-1}\right) .
$$

The vertex is 


$$
\mathcal{V}\left(g_{i j}\right)=\frac{1}{5 !} \int d \beta_{i} d h_{i j} \prod_{i<j} \delta\left(g_{j i}^{-1} h_{i j} \beta_{i}^{-1} \beta_{j} h_{j i} g_{i j}\right) .
$$

Notice that the kinetic operator $\mathcal{K}$ is not a projector anymore (i.e., $\mathcal{K}^{2} \neq \mathcal{K}$ ). As a consequence, the propagator $\mathcal{P}=\mathcal{K}^{-1}$ does not have a simple form in coordinate space.

If we expands the action (14) in terms of irreducible representations using the Peter-Weyl theorem, then the amplitude of a given Feynman diagram $J$ is given by

$$
A(J)=\sum_{c} \prod_{f} \operatorname{dim}\left(c_{f}\right) \prod_{v} \mathcal{B}\left(c_{v}\right),
$$

where the sum is now only over simple representations of $S O(4)$, and $\mathcal{B}\left(c_{v}\right)$ denotes Barrett-Crane vertex amplitude. This is given by the $15 j$-symbol constructed out of the 10 colors of the surfaces meeting at the vertex using the Barrett-Crane intertwiners. See (A7) and ( 23]).

\section{The new model}

The idea at the basis of the new model is the same as in Barrett-Crane: to modify the BF action (5) by inserting the projector (13) which implements the restriction of BF to GR. However, this time we insert the projection in the interaction term only, keeping the same kinetic term as in the BF theory. That is, we define the new model by the action

$$
S[\phi]=\int d g_{i}\left[P_{g} \phi\left(g_{i}\right)\right]^{2}+\frac{\lambda}{5 !} \int d g_{i}\left[P_{g} P_{h} \phi\left(g_{i}\right)\right]^{5}
$$

or

$$
S[\phi]=\int d g_{i}\left[P_{g} \phi\left(g_{i}\right)\right]^{2}+\frac{\lambda}{5 !} \int d g_{i}\left[P_{g} P_{h} P_{g} \phi\left(g_{i}\right)\right]^{5} .
$$

where $P_{h}$ and $P_{g}$ are defined in (6), and (13) respectively. As for the Barrett-Crane model, if we drop $P_{h}$ from the previous action we obtain the TOCY topological model of section (II). As we show below, the two forms of the action define the same theory, since the extra $P_{g}$ in the second expression can be always absorbed into the $P_{g}$ of some propagator when computing an amplitude. The second form of the action simplifies the analysis of the theory in momentum space.

From (19) the kinetic operator becomes

$$
\mathcal{K}\left(g_{i}, \tilde{g}_{i}\right)=\int d \gamma d h_{i} \prod_{i} \delta\left(g_{i} \gamma \tilde{g}_{i}^{-1}\right),
$$

which corresponds to the projector into the space of gauge invariant fields $\left(\mathcal{K}^{2}=\mathcal{K}\right)$. In this space its inverse is itself and the propagator of the theory is simply $\mathcal{P}=\mathcal{K}$ as in the TOCY model. The vertex of the theory is

$$
\mathcal{V}\left(g_{i j}\right)=\frac{1}{5 !} \int d \beta_{i} d \hat{\beta}_{i} d h_{i j} \prod_{i<j} \delta\left(g_{j i}^{-1} \hat{\beta}_{i} h_{i j} \beta_{i}^{-1} \beta_{j} h_{j i} \hat{\beta}_{j}^{-1} g_{i j}\right) .
$$

The $\beta$ and $\hat{\beta}$ integration variables correspond to the two projectors $P_{g}$ in the interaction. In the following we will show that the $\hat{\beta}$ integration is redundant when computing any amplitude, and therefore the two expressions in (19) and (20) define the same theory. The key feature of this model is that, as we will argue in the following, it does not contain divergences associated to the sum over colors for fundamental bubble diagrams.

\section{BUBBLE AMPLITUDES}

\section{A. Fundamental bubbles and Pachner moves}

As mentioned in the introduction, and discussed in detail in Ref. [27, in a spin foam model divergences arise in Feynman diagrams containing bubbles, that is, closed surfaces. Bubbles are the spin foam analog of the loop 
diagrams of standard QFT. To begin the analysis, we consider here only 2-complexes that are (the two skeleton of the) dual of regular triangulations. Therefore, we will talk equivalently about triangulations or 2-complexes.

We define a fundamental bubble diagram as a bubble diagram obtained from an elementary diagram without bubbles by means of the basic $4 \mathrm{~d}$ Pachner moves on the corresponding triangulation. These are the simplest diagrams presenting bubbles, and, in this sense, fundamental bubble diagrams are analogous to one-loop diagrams in standard QFT, representing the basic potentially divergent amplitudes in the model.

In four dimensions there are 3 possible Pachner moves -the 1-5, the 2-4, and the 3-3 Pachner moves respectivelyplus their inverses. Only the 1-5 and the 2-4 Pachner moves generate bubbles. In terms of a $4 \mathrm{~d}$ triangulation, the 1-5 move is defined by the split of a 4-simplex into five 4-simplices. More precisely, we put a point $p$ in the interior of the 4 -simplex with vertices $p_{i}, i=1, \ldots 5$, we add the five segments $\left(p, p_{i}\right)$, the ten triangles $\left(p, p_{i}, p_{j}\right)$, the ten tetrahedra $\left(p, p_{i}, p_{j}, p_{k}\right)$, and the five 4 -simplices $\left(p, p_{i}, p_{j}, p_{k}, p_{l}\right)$ (where $\left.i \neq j \neq k \neq l\right)$.

In the 2-4 move, we replace the two 4-simplexes $\left(a, p_{1}, p_{2}, p_{3}, p_{4}\right)$, and $\left(b, p_{1}, p_{2}, p_{3}, p_{4}\right)$, sharing the tetrahedron $\left(p_{1}, p_{2}, p_{3}, p_{4}\right)$ with the four 4 -simplices $\left(a, b, p_{i}, p_{j}, p_{k}\right)$ where $i \neq j \neq k=1, \ldots 4$.

In terms of the 2-complex (the 2-skeleton of the dual of the triangulation) which represent Feynman diagrams of our field theory, this set of moves generate the two fundamental bubble diagrams. The 1-5 move in the dual picture is illustrated in Fig. 3. We denote the diagram on the right as the 5-bubble diagram. The vertices of the picture are dual to the 4-simplexes of the triangulation; the edges of the picture are dual to the tetrahedra of the triangulation; the surfaces (in fact, here, all the triangles) are dual to the triangles of the triangulation.

The amplitude of a closed diagram is a number. The amplitude of an open diagram, that is, a diagram with a boundary, is a function of the variables on the boundary, as for conventional QFT Feynman diagrams. The boundary of a 2-complex is given by a graph, where the nodes are generated by the intersections of the edges with the boundary, and the links are generated by the intersections of the surfaces with the boundary. To start with, the amplitude of the open diagram is a function of 4 group arguments per each external line. However, consider a surface of an open 2-complex and the link $a b$ of the boundary graph that bounds it. Let $a$ and $b$ be the nodes on the boundary graph that bound $a b$. The surface determines a sequence of delta functions that starts with one of the group elements in $a$, say $g_{a}$ and ends with one of the group elements in $b$, say $g_{b}$. By integrating internal variables all these delta functions can be contracted to a single delta function of the form $\delta\left(g_{a} \ldots g_{b}^{-1}\right)$. This is of course a function of $g_{b}^{-1} g_{a}$. We can thus define the group variable $\rho_{a b}=g_{b}^{-1} g_{a}$, naturally associated to the link $a b$, and conclude that the amplitude of an open 2-complex is a function $A\left(\rho_{a b}\right)$ of one group element per each link of its boundary graph. In "momentum space", the amplitude of the diagram is a function over the possible colorings, in the sense of the spin networks, of the boundary graph. That is, if $s$ is a spin network given by a coloring of the boundary graph,

$$
A(s)=\int d \rho_{a b} \psi_{s}\left(\rho_{a b}\right) A\left(\rho_{a b}\right)
$$

where $\psi_{s}\left(\rho_{a b}\right)$ is the spin network function [12]. In Fig. 3, the thin lines in the picture represent the boundary graph of the diagram, that is the intersection of the 2 -complex with a 3 -sphere that bounds it. This intersection is a graph on the 3-sphere. Notice that the boundary remains the same after the move is implemented. We can think of the diagram on the right as a radiative correction to the diagram on the left. The analogous picture is shown in Fig. 1 for the 2-4 Pachner move. We denote the diagram on the right of Fig. 1 as the 1-bubble diagram.
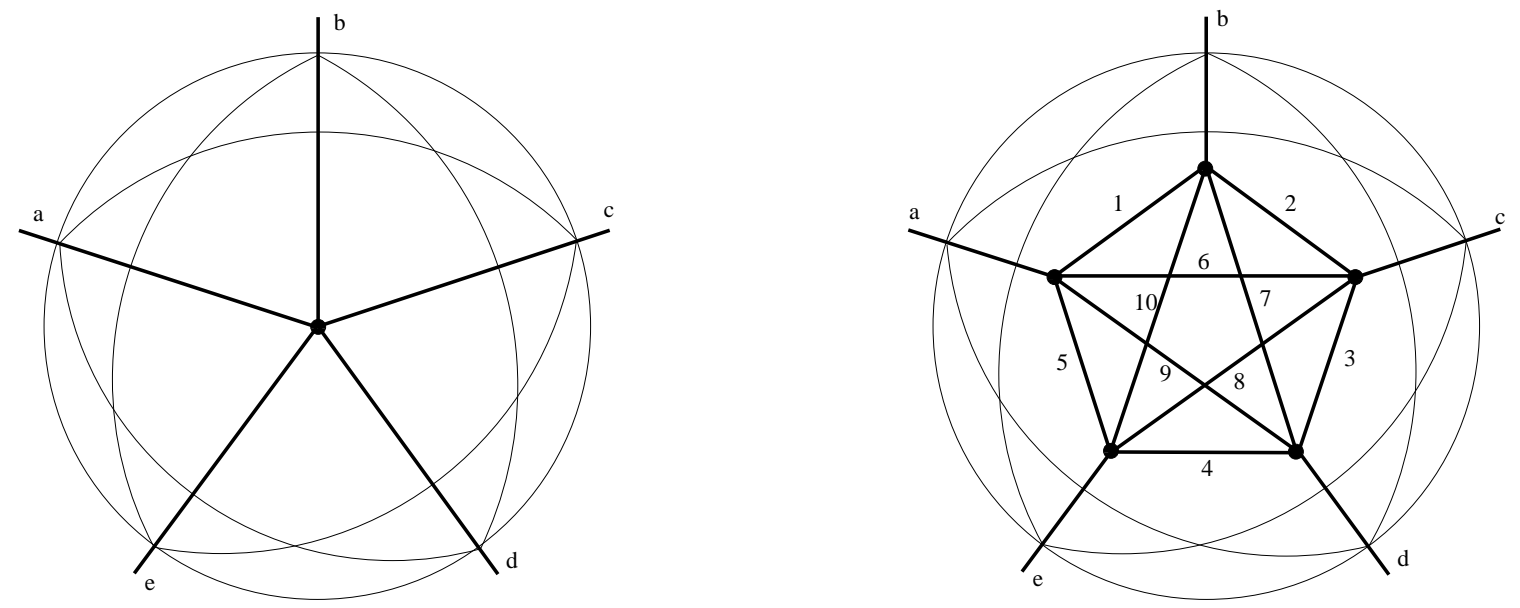

FIG. 3. The 1-5 Pachner move, on the right the fundamental 5-bubble Feynman diagram. 

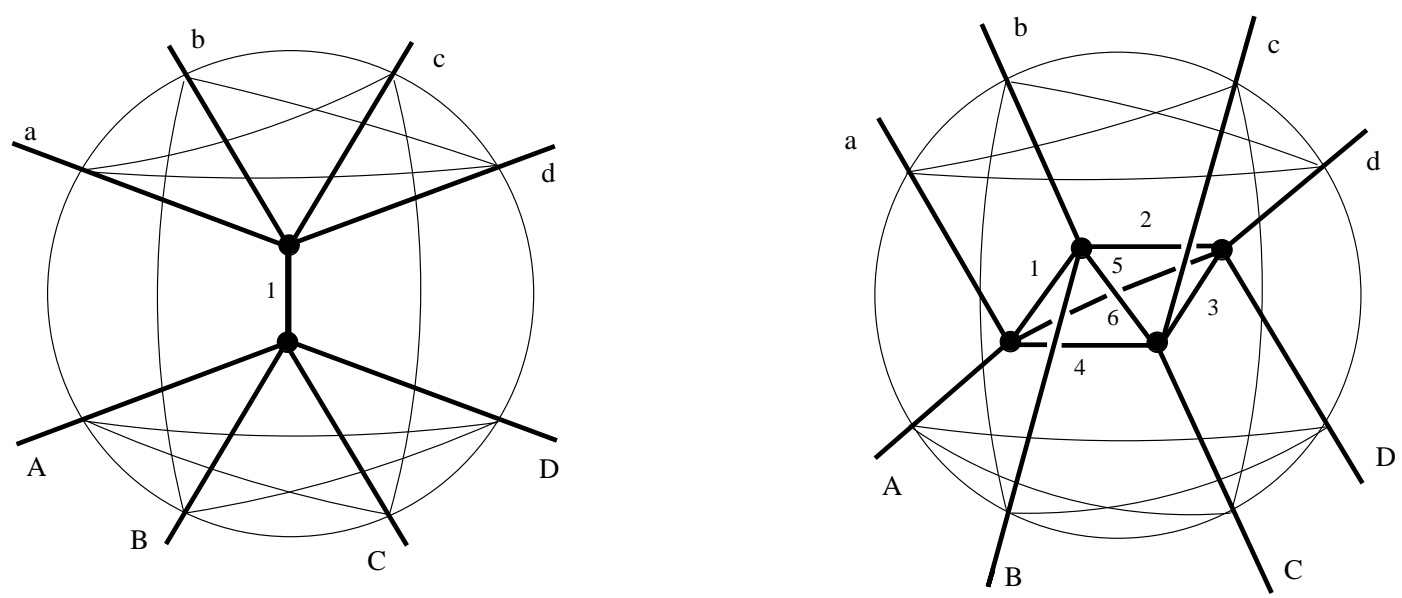

FIG. 4. The 2-4 Pachner move, on the right the fundamental 1-bubble Feynman diagram.

A straightforward calculation shows that the amplitudes of these diagrams diverge in the cases of the TOCY and Barrett-Crane model [27]. In the following section, we compute the amplitudes of the 5 and 1-bubbles.

\section{B. The 5-bubble amplitude}

We start by computing the amplitude $A_{5}$ corresponding to the diagram on the right of Fig. 3. We use the second form of the action (20) and show that the result is independent of this choice. The amplitude is a function of the group elements on the external propagators. It is obtained by writing the five vertices and the ten propagators in the diagram, and integrating on each propagator-vertex contraction. Notice that we are computing the amplitude of a fixed 2-complex, and therefore the permutation in (8) is already fixed, and we do not have to sum over permutations.

Let us introduce some notation. We label the five vertices in Fig. 3 by means of an index $a$ that takes values from 1 to 5. The ten internal edges can then be labeled by unordered couples $(a b)$ of indices. For notational convenience we label as $(0 a)$ the external edge emanating from the vertex $a$. Consider the $a$ vertex. It is a function of twenty group elements, four per leg, naturally paired by being in the same delta function, or, equivalently, by relating to the same 2-complex surface. Consider the leg $(a b)$ of the vertex $a$. We denote the group elements on this leg which is paired with the leg $(a c)$ as $g_{(a b)(a c)}$. Notice that the first couple of indices refer to the leg on which the group sits, while the second refers to the leg to which it is paired. The four group elements on external lines are denoted as $g_{(0 a)(a b)}$, while the group element on, say, the $(a b)$ edge and paired to the external edge is denoted $g_{(a b)(a 0)}$. Since there is one $h$ integration variable corresponding to each of the configuration variables $g_{(a b)(b c)}$, we label them according to the same rule. We denote the $\gamma$ integration variables in (21) associated to propagators connecting the vertex $a$ with the vertex $b$ as $\gamma_{a b}$ respectively. Given a vertex there is one $\beta$ and one $\hat{\beta}$ per leg corresponding to the two $P_{g}$ 's in the interaction (see (20)). Therefore, the $\beta$ integration variables in (22) are denoted as $\beta_{a(a b)}$, where the first index denotes the vertex to which $\beta$ belongs, while the second couple of indices denote the corresponding leg. The amplitude is then given by

$$
A_{5}\left(g_{a(a b)}\right)=\int[d g] \prod_{b} \mathcal{V}\left(g_{(c b)(b d)}\right) \prod_{c d} \mathcal{P}\left(g_{(c d)(c e)}, g_{(c d)(d e)}\right),
$$

where $[d g]$ denotes the integration over internal group elements (namely, $g_{(a b)(b c)}$ with $a, b \neq 0$ ), while the indices $c, d, e$ in the vertices and propagators can take the value 0 as well. As argued in the last section, the $g_{(0 a)(a b)}$ 's enter this expression only in the combination $\rho_{a b}=g_{(0 a)(a b)}^{-1} g_{(0 b)(a b)}$.

We have to insert the values (22) of the vertex amplitude and the value (8.9) of the propagator (without the sum over permutations) in this expression. Explicitly, and with the correct index structure we need, these are

$$
\mathcal{V}\left(g_{(c b)(b d)}\right)=\frac{1}{5 !} \int[d \beta][d \hat{\beta}][d h] \prod_{(c b)<(b d)} \delta\left(g_{(b d)(c b)}^{-1} \hat{\beta}_{b(c b)} h_{(c b)(b d)} \beta_{b(c b)}^{-1} \beta_{b(b d)} h_{(b d)(c b)} \hat{\beta}_{b(b d)}^{-1} g_{(c b)(b d)}\right)
$$

and 


$$
\mathcal{P}\left(g_{(c d)(c e)}, g_{(c d)(d e)}\right)=\int[d \gamma] \prod_{e=0, \ldots, 5, e \neq c, e \neq d} \delta\left(g_{(c d)(c e)} \gamma_{c d} \tilde{g}_{(c d)(d e)}^{-1}\right),
$$

where $[d \beta],[d \hat{\beta}],[d h]$, and $[d \gamma]$ denote integration over all the $\beta_{a(a b)}, \hat{\beta}_{a(a b)}, h_{(a b)(b c)}$, and $\gamma_{a b}$ variables respectively. We recall that all $h$ 's are in $S O(3)$, while all $\beta$ 's, $\gamma$ 's, $g$ 's, and $\rho$ 's are in $S O(4)$. Inserting (25) and (26) in (24) we obtain a complicated multiple integral of delta functions, which we now analyze by breaking it into pieces.

The key observation is that the delta functions appear in sequences, corresponding to the boundaries of the surfaces of the faces of the 2-complex, or to the connected lines obtained by replacing vertices and propagators in Fig. 3 with the vertex and propagators given in Fig. 1 and 2. It is easy to see that there are two kinds of such sequences, corresponding to the two kind of surfaces in the 2-complex. These are illustrated in Fig. 5.
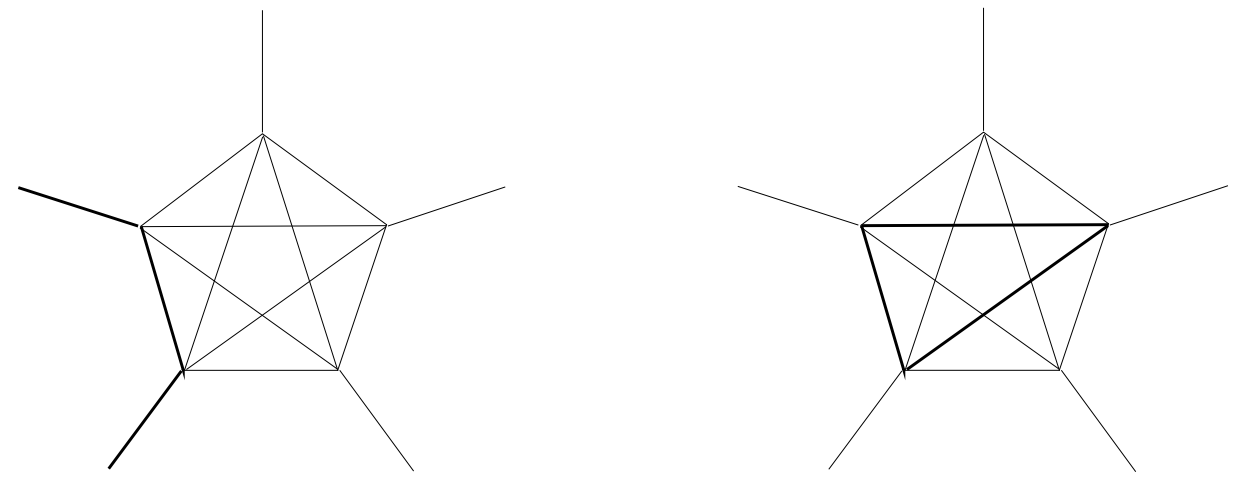

FIG. 5. Two possible terms contributing to the $A_{5}$ amplitude

The terms of the first kind follow the pattern shown in the diagram on the left of Fig. 5; we denote them as wedges. The ten terms of the second kind have the form of the diagram shown on the right, and we denote them as faces. There are ten terms of the first kind and ten terms of the second kind. Consider the term of the first kind. Let $(a b)$ be the link in this term. From the definitions of the vertex and propagator respectively we have that the corresponding sequence of delta functions is

$$
\begin{array}{r}
\delta\left(g_{(0 a)(a b)} \hat{\beta}_{a(0 a)} h_{(0 a)(a b)} \beta_{a(0 a)}^{-1} \beta_{a(a b)} h_{(a b)(0 a)} \hat{\beta}_{a(a b)}^{-1} g_{(a b)(0 a)}^{-1} \delta\left(g_{(a b)(0 a)} \gamma_{(a b)} g_{(a b)(0 b)}^{-1}\right)\right. \\
\delta\left(g_{(a b)(0 b)} \hat{\beta}_{b(a b)} h_{(0 b)(a b)} \beta_{b(a b)}^{-1} \beta_{b(0 b)} h_{(0 b)(a b)} \hat{\beta}_{b(0 b)}^{-1} g_{(b 0)(a b)}^{-1}\right),
\end{array}
$$

where the first and the third delta functions come from the vertex $a$ and the vertex $b$ respectively, and the delta in the middle comes from one of the four deltas in the propagator $a b$ connecting the two vertices. By integrating over the two variables that concatenate the delta functions, namely $g_{(a b)(0 a)}$ and $g_{(a b)(0 b)}$, we obtain the quantity

$$
E_{a b} \equiv \delta\left(\hat{\beta}_{b(a b)} h_{(a b)(0 b)} \beta_{b(a b)}^{-1} \beta_{b(0 b)} h_{(0 b)(a b)} \beta_{b(0 b)}^{-1} \rho_{b a} \hat{\beta}_{a(0 a)} h_{(0 a)(a b)} \beta_{a(0 a)}^{-1} \beta_{a(a b)} h_{(a b)(0 a)} \hat{\beta}_{a(a b)}^{-1} \gamma_{a b}\right),
$$

We have rearranged the argument of the delta function using that $\delta\left(g_{1} g_{2}\right)=\delta\left(g_{2} g_{1}\right)$, and we have defined $\rho_{a b} \equiv$ $g_{(0 a)(a b)}^{-1} g_{(0 b)(a b)}$.

The second kind of terms, illustrated on the right of Fig. IIIB, come from the ten internal faces in the 5-bubble diagram. Consider the face bounded by the three vertices $a b c$. The corresponding term gives the sequence of deltas

$$
\begin{gathered}
\delta\left(g_{(a c)(a b)} \hat{\beta}_{a(c a)} h_{(a c)(a b)} \beta_{a(c a)}^{-1} \beta_{a(a b)} h_{(a b)(a c)} \hat{\beta}_{a(a b)}^{-1} g_{(a b)(a c)}^{-1}\right) \delta\left(g_{(a b)(a c)} \gamma_{(a b)} g_{(a b)(b c)}^{-1}\right) \\
\delta\left(g_{(a b)(b c)} \hat{\beta}_{b(a b)} h_{(a b)(b c)} \beta_{b(a b)}^{-1} \beta_{b(b c)} h_{(b c)(a b)} \hat{\beta}_{b(b c)}^{-1} g_{(b c)(a b)}^{-1} \delta\left(g_{(b c)(a b)} \gamma_{(b c)} g_{(b c)(a c)}^{-1}\right)\right. \\
\delta\left(g_{(b c)(a c)} \hat{\beta}_{c(b c)} h_{(b c)(a c)} \beta_{c(b c)}^{-1} \beta_{(c) c a} h_{(a c)(b c)} \hat{\beta}_{c(c a)}^{-1} g_{(a c)(b c)}^{-1} \delta\left(g_{(a c)(b c)} \gamma_{(a c)} g_{(a c)(a b)}^{-1}\right)\right.
\end{gathered}
$$

Again, we can integrate over the intermediate group elements, $g_{(a c)(a b)}, g_{(a b)(a c)}, g_{(a b)(b c)}, g_{(b c)(a b)}, g_{(b c)(a c)}$, and $g_{(a c)(b c)}$ obtaining

$$
\begin{aligned}
& F_{a b c} \equiv \delta \underbrace{\beta_{a(a b)} h_{(a b)(a c)} \hat{\beta}_{a(a b)}^{-1} \gamma_{(a b)} \hat{\beta}_{b(a b)} h_{(a b)(b c)} \beta_{b(a b)}^{-1}}_{\rho_{a b}^{a b c}} \beta_{b(b c)} h_{(b c)(a b)} \hat{\beta}_{b(b c)}^{-1} \\
&\left.\gamma_{(b c)} \hat{\beta}_{c(b c)} h_{(b c)(a c)} \beta_{c(b c)}^{-1} \beta_{c(c a)} h_{(a c)(b c)} \hat{\beta}_{c(c a)}^{-1} \gamma_{(a c)} \hat{\beta}_{a(c a)} h_{(a c)(a b)} \beta_{a(c a)}^{-1}\right),
\end{aligned}
$$


Notice that the $\gamma_{a b}$ 's associated to the internal propagators appear surrounded by $\hat{\beta}$ 's in the same way in $E_{a b}$ and in $F_{a b c}$; therefore we can reabsorb all the $\hat{\beta}$ 's by redefining the integration variables $\gamma_{a b}$ (that is $\gamma_{a b} \rightarrow \hat{\beta}_{(a) a b}^{-1} \gamma_{a b} \hat{\beta}_{(b) a b}$ ) using the right-left invariance of the Haar measure. This shows that the $P_{g}$ on the right in the interaction term of the action (20) is redundant and that the two forms (19) and (20) of the action are equivalent.

Using the definitions (28) and (29), we can rewrite the amplitude (24) as

$$
A_{5}\left(\rho_{a b}\right)=\int[d \gamma][d \beta][d \hat{\beta}][d h] \prod_{a b} E_{a b} \prod_{a b c} F_{a b c} .
$$

We can eliminate the $E_{a b}$ 's by integrating over the $\gamma_{a b}$ 's. This gives

$$
\gamma_{a b}=\hat{\beta}_{a(a b)} h_{(a b)(0 a)} \beta_{a(a b)}^{-1} \beta_{a(0 a)} h_{(0 a)(a b)} \hat{\beta}_{a(0 a)}^{-1} \rho_{a b} \hat{\beta}_{b(0 b)} h_{(0 b)(a b)} \beta_{b(0 b)}^{-1} \beta_{b(a b)} h_{(a b)(0 b)} \hat{\beta}_{b(a b)}^{-1},
$$

We substitute these values into the remaining deltas; we define (see the emphasized term in equation (29))

$$
\rho_{a b}^{a b c}=\beta_{a(a b)} h_{(a b)(a c)} h_{(a b)(0 a)} \beta_{a(a b)}^{-1} \beta_{a(0 a)} h_{(0 a)(a b)} \hat{\beta}_{a(0 a)}^{-1} \rho_{a b} \hat{\beta}_{b(0 b)} h_{(0 b)(a b)} \beta_{b(0 b)}^{-1} \beta_{b(a b)} h_{(a b)(0 b)} h_{(a b)(b c)} \beta_{b(a b)}^{-1} .
$$

We can redefine the $h_{(a b)(b c)}$ variables, by absorbing the $h_{(a b)(0 b)}$ into them. We substitute these expression in the $F$ 's, and the amplitude becomes

$$
A_{5}\left(\rho_{a b}\right)=\int[d \beta][d \hat{\beta}][d h] \prod_{a b c} \delta\left(\rho_{a b}^{a b c} \rho_{b c}^{a b c} \rho_{c a}^{a b c}\right) .
$$

In order to show that $A_{5}\left(\rho_{a b}\right)$ is finite, it is sufficient to show that the "vacuum" value of the amplitude is finite [27. In momentum space this is the value of the amplitude when the colors of the boundary lines are zero. In configuration space this value is obtained by integrating the amplitude (34) over $\rho_{a b}$ (Integration over the external group variables projects the amplitude to the trivial representation). We want thus to show that

$$
\mathcal{I}_{5}=\int[d \rho] A_{5}\left(\rho_{a b}\right)<\infty
$$

The amplitude when the external colors are not all zero is given by the integral of $A_{5}^{(b c)}(\rho)$ times the appropriate spin-network state, which is a bounded function over the group. The result of this integration is finite if (35) holds.

If we insert (33) and (34) into (35), we see that several of the variables can be absorbed into redefinitions of integration variables. In particular, we can redefine $\rho_{a b} \rightarrow \beta_{a(0 a)} h_{(0 a)(a b)} \hat{\beta}_{a(0 a)}^{-1} \rho_{a b} \hat{\beta}_{b(0 b)} h_{(0 b)(a b)} \beta_{b(0 b)}^{-1}$ in (32), and obtain the following simpler expression

$$
\begin{aligned}
\mathcal{I}_{5}=\int[d \rho][d \beta][d h] & \prod_{a b c} \delta\left(\beta_{a(a b)} h_{(a b)(b c)} \beta_{a(a b)}^{-1} \rho_{a b} \beta_{b(a b)} h_{(c b)(a b)} \beta_{b(a b)}^{-1}\right. \\
& \left.\beta_{b(b c)} h_{(a b)(b c)} \beta_{b(b c)}^{-1} \rho_{b c} \beta_{c(b c)} h_{(b c)(a b)} \beta_{c(b c)}^{-1} \beta_{c(c a)} h_{(c a)(c b)} \beta_{c(c a)}^{-1} \rho_{c a} \beta_{a(c a)} h_{(c b)(c a)} \beta_{a(c a)}^{-1}\right) .
\end{aligned}
$$

We can write this as

$$
\mathcal{I}_{5}=\int[d \rho][d \beta][d h] \prod_{a b c} \delta\left(\tilde{\rho}_{a b}^{a b c} \tilde{\rho}_{b c}^{a b c} \tilde{\rho}_{c d}^{a b c}\right)
$$

where

$$
\tilde{\rho}_{a b}^{a b c}=\beta_{(a) a b} h_{(a b)(b c)} \beta_{(a) a b}^{-1} \rho_{a b} \beta_{(b) a b} h_{(b c)(a b)} \beta_{(b) a b}^{-1} .
$$

Now, in order to simplify this integral, we observe that the integrand in (36) is gauge invariant under the gauge transformation $\rho_{a b} \rightarrow \mu_{a} \rho_{a b} \mu_{b}^{-1}$ with $\mu_{a} \in S O(4)$, since the $\mu_{a}$ 's can be absorbed by redefining the $\beta$ 's. The integral is therefore equal to the gauge fixed integral at a particular gauge, times the volume of the gauge orbit (which is unity since we are working with the normalized Haar measure). We fix the gauge by requiring $\rho_{12}=\rho_{13}=\rho_{14}=\rho_{15}=1$. Taking this into account, we can integrate away the variables $\rho_{\hat{b} \hat{c}}$, where an index with hat takes the values 2 to 5 only. The delta functions -associated to faces in which 2 of the $3 \rho$ 's have been gauge fixed- fix the value of these variables to 


$$
\begin{aligned}
\rho_{\hat{b} \hat{c}}=\beta_{\hat{b}(\hat{b} \hat{c})} h_{(\hat{b} \hat{c})(\hat{c} 1)} \beta_{\hat{b}(\hat{b} \hat{c})}^{-1} \beta_{\hat{b}(1 \hat{b})} h_{(\hat{b} \hat{c})(1 \hat{b})} \beta_{\hat{b}(1 \hat{b})}^{-1} \beta_{1(1 \hat{b})} \hat{h}_{(1 \hat{b})(\hat{b} \hat{c})} \beta_{1(1 \hat{b})}^{-1} & \\
& \beta_{1(\hat{c} 1)} h_{(\hat{c} 1)(1 \hat{b})} \beta_{1(\hat{c} 1)}^{-1} \beta_{\hat{c}(\hat{c} 1)} h_{(1 \hat{b})(\hat{c} 1)} \beta_{\hat{c}(\hat{c} 1)}^{-1} \beta_{\hat{c}(\hat{b} \hat{c})} h_{(\hat{c} 1)(\hat{b} \hat{c})} \beta_{\hat{c}(\hat{b} \hat{c})}^{-1} .
\end{aligned}
$$

Inserting this in (38), the external $\beta^{\prime} s$ simplify and two $h$ ' can be collapsed into one by redefinition. There remain only the four delta functions associated to the faces $\hat{a} \hat{b} \hat{c}$. Each $h$ variable turns out to be sandwiched between $\beta$ and $\beta^{-1}$. Thus, integrating in such an $h$ variable is indeed integrating over the subgroup $\beta H$ of $S O(4)$, formed by the elements that leave $\beta \hat{t}$ invariant. Taking all this into account, we conclude

$$
\mathcal{I}_{5}=\int d \beta_{b(c d)} \int_{\beta_{(a b)(c d)} H} d \mathfrak{h}_{(a b)(c d)} \prod_{a b c=2, \ldots, 5} \delta\left(\tilde{\rho}_{a b}^{a b c} \tilde{\rho}_{b c}^{a b c} \tilde{\rho}_{c d}^{a b c}\right),
$$

with

$$
\tilde{\rho}_{a b}^{a b c}=\mathfrak{h}_{(b c)(c d)} \mathfrak{h}_{(a b)(b c)} \mathfrak{h}_{(a b)(a c)} \mathfrak{h}_{(c a)(a b)} \mathfrak{h}_{(a c)(b d)} \mathfrak{h}_{(b c)(b d)},
$$

where $\mathfrak{h}_{(a b)(b c)} \in \beta_{(a b)(b c)} H$ (each $\mathfrak{h}$ corresponds to a different $S O(3)$ subgroup).

As a check, imagine now that we are dealing with BF theory. To obtain BF theory, we have simply to drop the integrations over $h$ 's. The resulting divergence is immediately $(\delta(1))^{4}$, which is the correct divergent factor for the 1-5 pachner move in BF theory [18,27]. Does the integrations over the subgroup $H$ absorb all the divergences? If it wasn't for the integration of the $\beta$ 's, the answer would be no, because we would simply obtain a divergence proportional to the fourth power of the value on the origin of the delta on $G / H$. However, the combined integration over the $h$ 's and the $\beta$ 's is sufficient to absorb all the divergences.

In fact, the integral we are studying is over a compact domain. Therefore divergences can only come from particular points where the integrand diverges. The point where the most serious divergences can appear is the origin $\beta_{(a b)(c d)}=h_{(a b)(c d)}=1$. Let us study the behavior of the integral around this point. To this aim, it is sufficiently to study an arbitrarily small neighborhood of this point. On an arbitrarily small neighborhood of the origin, we can describe the group by means of its algebra. We can thus replace the group integral with an integral over the algebra. To the order we are interested, we can take the Lebesgue measure over the algebra, since the Jacobian between this measure and the measure induced by the Haar measure on the group goes smoothly to 1 on the origin. Group elements can be written as exponents of algebra elements, and products of group elements can be expanded to first order around the origin, giving commutators in the algebra. The four $S O(4)$ delta functions give 24 1-dimensional deltas. It is lengthy (because of the many variables), but completely straightforward, to see that the 24 resulting 1-dimensional delta functions in the previous expression are not redundant and therefore the value of the amplitude is finite. We will present the details of the calculation elsewhere.

A simple proof of the finiteness of the previous amplitude will be given in terms of the mode expansion in momentum space.

\section{The 1-bubble amplitude}

We now consider the amplitude of the diagram on the right of Fig. 1 . This is another divergent amplitude in BF theory and in the Barrett-Crane model. We now show that the amplitude is finite in the new model. The pattern for analyzing this amplitude is the same as in the previous case, but simpler. We only sketch here the key steps of the calculation, leaving the details to the reader. Integration over the six upper external wedges (see Fig. 价) implies, in analogy to the previous case, that

$$
\gamma_{a b}=\hat{\beta}_{a(a b)} h_{(a b)(0 a)} \beta_{a(a b)}^{-1} \beta_{a(0 a)} h_{(0 a)(a b)} \hat{\beta}_{a(0 a)}^{-1} \rho_{a b} \hat{\beta}_{b(0 b)} h_{(0 b)(a b)} \beta_{b(0 b)}^{-1} \beta_{b(a b)} h_{(a b)(0 b)} \hat{\beta}_{b(a b)}^{-1} .
$$

substituting this into the remaining deltas we obtain (schematically)

$$
\begin{array}{r}
A_{4}(\rho)=\int[d \beta][d h] \prod_{a b A B} \delta\left(\beta_{a(a b)} h_{(B A)(0 A)} \beta_{a(a b)}^{-1} \beta_{a(0 a)} h_{(0 a)(a b)} \rho_{a b} h_{(0 b)(a b)} \beta_{b(0 b)}^{-1}\right. \\
\left.\beta_{b(a b)} \hat{h}_{(B A)(0 B)} \beta_{b(a b)}^{-1} \beta_{b(0 B)} h_{(0 B)(B A)} \rho_{B A} h_{(0 A)(B A)} \beta_{a(0 A)}^{-1}\right) \\
\prod_{a b c} \delta\left(\rho_{a b}^{(a b c)} \rho_{b c}^{(a b c)} \rho_{c a}^{(a b c)}\right) \prod_{a A} \delta\left(\beta_{a(0 a)} h_{(0 a)(0 A)} \rho_{a A} h_{(0 A)(0 a)} \beta_{a(0 A)}^{-1}\right),
\end{array}
$$


Now we want to show that the "vacuum bubble" is finite, namely:

$$
\mathcal{I}_{4}=\int[d \rho] A_{4}(\rho)<\infty
$$

Integrating over $\rho_{A B}$ and $\rho_{a A}$ this reduces to

$$
\mathcal{I}_{4}=\int[d \rho][d \beta][d h] \prod_{a b c} \delta\left(\rho_{a b}^{(a b c)} \rho_{b c}^{(a b c)} \rho_{c a}^{(a b c)}\right) .
$$

As in the previous case we can fix the gauge by means of the conditions $\rho_{12}=\rho_{13}=\rho_{14}=1$. We then integrate over the remaining $\rho$ 's which eliminate three of the four delta functions, and fix the value of the remaining $\rho_{c d}$ (for $c, d \neq 1)$ to

$$
\begin{aligned}
\rho_{b c}=\beta_{b(b c)} h_{(b c)(1 b)} \beta_{b(b c)}^{-1} \beta_{b(a b)} h_{(a b)(a c)} \beta_{b(a b)}^{-1} \beta_{a(a b)} h_{(a b)(b c)} \beta_{a(a b)}^{-1} & \\
& \beta_{a(c a)} h_{(c a)(a b)} \beta_{a(c a)}^{-1} \beta_{c(c a)} h_{(c a)(c b)} \beta_{c(c a)}^{-1} \beta_{c(b c)} h_{(b c)(c a)} \beta_{c(b c)}^{-1} .
\end{aligned}
$$

Finally, $\mathcal{I}_{4}$ reduces to an integral of single delta function. This integral is the integration of the distribution $\mathcal{D}\left(g_{1}, g_{2}, g_{3}\right)$ defined in (A8). By lemma(1) in the appendix, the integral is finite.

\section{SPIN FOAM FORMULATION}

Using Peter-Weyl theorem, we can analyze the new model in momentum space. This analysis explicitly connects our results to the spin foam formalism. In particular, we show that the vertex amplitude of the theory is essentially the same as the one in the Barrett-Crane model, up to a rescaling, that can be viewed as an edge amplitude. We also present here another proof that the amplitudes calculated in the previous section are finite, and finally we present arguments supporting the conjecture that amplitudes are finite at any order.

According to the Peter-Weyl theorem, given an $\mathcal{L}^{2}[G]$ (Haar-square integrable) function $\phi(g)$ over the group $G=S O(4)$, we can expand it in terms of the matrices $D_{\alpha \beta}^{(\Lambda)}(g)$ of the irreducible representations $\Lambda$ of $S O(4)$; that is,

$$
\phi(g)=\sum_{\Lambda} \phi_{\alpha \beta}^{\Lambda} D_{\alpha \beta}^{(\Lambda)}(g) .
$$

We begin by analyzing the kinetic term in (19). Using (6) and equation (A4) we obtain

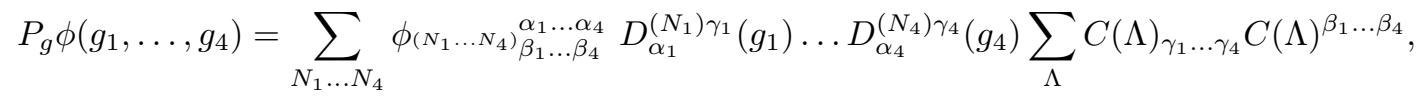

See the appendix for notation and definitions. In order to have a more compact notation, we have dropped the indices $N_{1} \ldots N_{4}$ from the expression for the intertwiners and we have kept only the color $\Lambda$ that labels them (namely we use $\left.C_{\gamma_{1} \ldots \gamma_{4}}^{N_{1} \ldots N_{4} \Lambda}=C(\Lambda)_{\gamma_{1} \ldots \gamma_{4}}\right)$. We use Einstein summation convention over repeated indices. There is no difference between upper or lower indices and we write them in a way or the other for notational convenience. We can simplify the previous expansion by defining the new field components

$$
\Phi_{N_{1} \ldots N_{4}, \Lambda}^{\alpha_{1} \ldots \alpha_{4}} \equiv \frac{\phi_{\left(N_{1} \ldots N_{4}\right)}^{\alpha_{1} \ldots \alpha_{4}} C(\Lambda)^{\beta_{1} \ldots \beta_{4}}}{\left(\Delta_{N_{1}} \ldots \Delta_{N_{4}}\right)^{\frac{3}{2}}}
$$

where $\Delta_{N}$ denotes the dimension of the irreducible representation of order $N$, and the factor $\left(\Delta_{N_{1}} \ldots \Delta_{N_{4}}\right)^{\frac{3}{2}}$ has been chosen to simplify the expression of the interaction vertex computed below. This choice of field yields the mode expansion:

$$
P_{g} \phi\left(g_{1}, \ldots, g_{4}\right)=\sum_{N_{1} \ldots N_{4}, \Lambda}\left(\Delta_{N_{1}} \ldots \Delta_{N_{4}}\right)^{\frac{3}{2}} \Phi_{N_{1} \ldots N_{4}, \Lambda}^{\alpha_{1} \ldots \alpha_{4}} D_{\alpha_{1}}^{\left(N_{1}\right) \gamma_{1}}\left(g_{1}\right) \ldots D_{\alpha_{4}}^{\left(N_{4}\right) \gamma_{4}}\left(g_{4}\right) C(\Lambda)_{\gamma_{1} \ldots \gamma_{4}} .
$$

Using (A1) and the orthonormality of the intertwiners, the kinetic term in (19) becomes

$$
\mathcal{K}=\sum_{N_{1} \ldots N_{4}, \Lambda} \Phi_{N_{1} \ldots N_{4}, \Lambda}^{\alpha_{1} \ldots \alpha_{4}} \Phi_{N_{1} \ldots N_{4}, \Lambda}^{\mu_{1} \ldots \mu_{4}}\left(\Delta_{N_{1}} \ldots \Delta_{N_{4}}\right)^{2} \delta_{\alpha_{1} \mu_{1}} \ldots \delta_{\alpha_{4} \mu_{4}}
$$


We can directly read the propagator of the theory from this expression

$$
\mathcal{P}_{\alpha_{1} \mu_{1} \ldots \alpha_{4} \mu_{4}}=\frac{\delta_{\alpha_{1} \mu_{1}} \ldots \delta_{\alpha_{4} \mu_{4}}}{\left(\Delta_{N_{1}} \ldots \Delta_{N_{4}}\right)^{2}} .
$$

In order to write the potential term we need to express $P_{g} P_{h} P_{g} \phi$ in terms of irreducible representations (see 19). Starting with (48) and using equations (A4) and (A5) we obtain

$$
\begin{aligned}
P_{g} P_{h} P_{g} \phi= & \sum_{N_{1} \ldots N_{4}, \Lambda}\left(\Delta_{N_{1}} \ldots \Delta_{N_{4}}\right)^{\frac{3}{2}} \Phi_{N_{1} \ldots N_{4}, \Lambda}^{\alpha_{1} \ldots \alpha_{4}} D_{\alpha_{1}}^{\left(N_{1}\right) \gamma_{1}}\left(g_{1}\right) \ldots D_{\alpha_{4}}^{\left(N_{4}\right) \gamma_{4}}\left(g_{4}\right) \\
& \sum_{N} C(N)_{\gamma_{1} \ldots \gamma_{4}} C(N)^{\beta_{1} \ldots \beta_{4}} w_{\beta_{1}} \ldots w_{\beta_{4}} w^{\mu_{1}} \ldots w^{\mu_{4}} C(\Lambda)_{\mu_{1} \ldots \mu_{4}} .
\end{aligned}
$$

Applying equation (A6) and (A7) we obtain

$$
P_{g} P_{h} P_{g} \phi=\sum_{N_{1} \ldots N_{4}, \Lambda} \sqrt{\Delta_{N_{1}} \ldots \Delta_{N_{4}}} \Phi_{N_{1} \ldots N_{4}, \Lambda}^{\alpha_{1} \ldots \alpha_{4}} D_{\alpha_{1}}^{\left(N_{1}\right) \gamma_{1}}\left(g_{1}\right) \ldots D_{\alpha_{4}}^{\left(N_{4}\right) \gamma_{4}}\left(g_{4}\right) B_{\gamma_{1} \ldots \gamma_{4}},
$$

where the sum is now over simple representations only, and $B_{\gamma_{1} \ldots \gamma_{4}}$ denotes the Barrett-Crane intertwiner. Using the previous equation the potential term in (19) becomes

$$
\frac{1}{5 !} \sum_{N_{1} \ldots N_{10}} \sum_{\Lambda_{1} \ldots \Lambda_{5}} \Phi_{N_{1} N_{2} N_{3} N_{4}, \Lambda_{1}}^{\alpha_{1} \alpha_{2} \alpha_{3} \alpha_{4}} \Phi_{N_{4} N_{5} N_{6} N_{7}, \Lambda_{2}}^{\alpha_{4} \alpha_{5} \alpha_{6} \alpha_{7}} \Phi_{N_{7} N_{3} N_{8} N_{9}, \Lambda_{3}}^{\alpha_{7} \alpha_{3} \alpha_{8} \alpha_{9}} \Phi_{N_{9} N_{6} N_{2} N_{10}, \Lambda_{4}}^{\alpha_{9} \alpha_{6} \alpha_{2} \alpha_{10}} \Phi_{N_{10} N_{8} N_{5} N_{1}, \Lambda_{5}}^{\alpha_{10} \alpha_{8} \alpha_{5} \alpha_{1}} \mathcal{B}_{N_{1}, \ldots, N_{10}}
$$

where $\mathcal{B}_{N_{1}, \ldots, N_{10}}$ corresponds to a $15 j$-symbol constructed with Barrett-Crane intertwiners which corresponds to the Barrett-Crane vertex amplitude [7]. Explicitly,

$$
\mathcal{B}_{N_{1}, \ldots, N_{10}}:=B_{\alpha_{1} \alpha_{2} \alpha_{3} \alpha_{4}}^{N_{1} N_{2} N_{3} N_{4}} B_{\alpha_{4} \alpha_{5} \alpha_{6} \alpha_{7}}^{N_{4} N_{5} N_{6} N_{7}} B_{\alpha_{7} \alpha_{3} \alpha_{8} \alpha_{9}}^{N_{7} N_{3} N_{8} N_{9}} B_{\alpha_{9} \alpha_{6} \alpha_{2} \alpha_{10}}^{N_{9} N_{6} N_{2} N_{10}} B_{\alpha_{10} \alpha_{8} \alpha_{5} \alpha_{1}}^{N_{10} N_{8} N_{5} N_{1}}
$$

Thus, the potential part of the action in the new model is given by (55) as in the Barrett-Crane model. Notice, however, that there is an extra sum over $\Lambda$ in (55), absent in Barrett-Crane. The propagator (52) of the theory in momentum space is rescaled with respect to the Barrett-Crane propagator $\left(\mathcal{P}_{\alpha_{1} \mu_{1} \ldots \alpha_{4} \mu_{4}}=\left(\Delta_{1} \ldots \Delta_{4}\right)^{-2} \mathcal{P}_{\alpha_{1} \mu_{1} \ldots \alpha_{4} \mu_{4}}^{(B C)}\right.$. As a consequence of this rescaling, and of the extra sum over $\Lambda$, there is a non-trivial amplitude associated to edges in the spin foam. Each edge contributes to the amplitude as

$$
A_{e}=\frac{\Delta_{N_{1}, \ldots, N_{4}}}{\left(\Delta_{N_{1}} \ldots \Delta_{N_{4}}\right)^{2}}
$$

where $N_{1}$ to $N_{4}$ are the colors of the four faces meeting at the given edge, and $\Delta_{N_{1}, \ldots, N_{4}}$ is the dimension of the space of the interwiners between the representations $N_{1}, \ldots, N_{4}$. In conclusion, the amplitude of a Feynman diagram $J$ is given by

$$
A(J)=\sum_{N} \prod_{f} \Delta_{N_{f}} \prod_{e} A_{e} \prod_{v} \mathcal{B}_{N_{1} \ldots N_{10}}
$$

where the sum is over simple representations $N$ of $S O(4)$, and $\mathcal{B}$ denotes the Barrett-Crane vertex amplitude.

Equivalently, as every edge connects two vertices, we can absorb the edge amplitude in the vertex amplitude and write $Z$ in the standard form (1), where the vertex amplitude is

$$
A_{v}=\frac{\prod_{i} \Delta_{N_{i 1}, \ldots, N_{i 4}}^{1 / 2}}{\left(\Delta_{N_{1}} \ldots \Delta_{N_{10}}\right)^{2}} \mathcal{B}_{N_{1} \ldots N_{10}},
$$

where $N_{1} \ldots N_{10}$ are the ten colors of the ten faces adjacent to the vertex $v$, and $N_{i 1} \ldots N_{i 4}, i=1 \ldots 5$ are the four colors of the four faces adjacent to $i$ 'th edge adjacent to the vertex $v$.

We close this section with a comment. Unlikely the Barrett-Crane model, in the mode expansion of the model presented here, the field depends on five representations (four external and one intertwiner), which can be seen precisely as the quantum numbers of a "first quantized" geometry of a tetrahedron. 


\section{A. Bubbles in the spin foam formulation}

Now we are ready to show the finiteness of the 5-bubble amplitude (equation (35) in the previous section) directly in momentum space. As we mentioned before, the integration over $\rho_{a b}$ in (35) projects the amplitude into the trivial representations. The value of $\mathcal{I}_{5}$ is then given by the sum over colors of the amplitudes corresponding to the diagram on the right of Fig. 3, in which the colors of the external faces are fixed to zero. Four of the colors vanish in each of the five vertices, and a typical vertex amplitude reduces to

$$
\mathcal{B}_{0000 N_{1} \ldots N_{6}}=\left\{\begin{array}{lll}
N_{1} & N_{2} & N_{3} \\
N_{4} & N_{5} & N_{6}
\end{array}\right\}_{6 j},
$$

where the RHS denotes the $6 J$-symbol defined in the appendix. Each of the internal faces is colored by irreducible representations $N_{1}$ to $N_{10}$. The internal faces are triangles; therefore for each $\Delta_{N}$, contribution of the face, there will

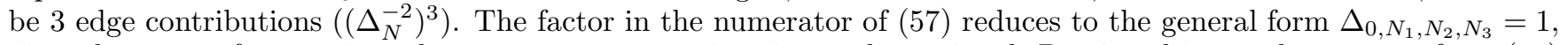
since the space of intertwiners between 3 representations is one dimensional. Putting this together, we get from (58)

$$
\mathcal{I}_{5}=\sum_{N_{1} \ldots N_{10}}\left(\Delta_{N_{1}} \ldots \Delta_{N_{10}}\right)\left(\Delta_{N_{1}} \ldots \Delta_{N_{10}}\right)^{-6} \prod_{v}\left\{\begin{array}{ccc}
N_{1}^{v} & N_{2}^{v} & N_{3}^{v} \\
N_{4}^{v} & N_{5}^{v} & N_{6}^{v}
\end{array}\right\}_{6 j} .
$$

Using the bound for the $6 j$-symbols given in (A12), and noticing that each color appears in three of the vertices, we conclude:

$$
\left|\mathcal{I}_{5}\right| \leq \sum_{N_{1} \ldots N_{10}}\left(\Delta_{N_{1}} \ldots \Delta_{N_{10}}\right)\left(\Delta_{N_{1}} \ldots \Delta_{N_{10}}\right)^{-6}\left(\Delta_{N_{1}} \ldots \Delta_{N_{10}}\right)^{3 / 2}=\sum_{N_{1} \ldots N_{10}}\left(\Delta_{N_{1}} \ldots \Delta_{N_{10}}\right)^{-\frac{7}{2}}<\infty
$$

Next, consider the case in which the external colors are not zero. The finiteness of these terms follows from lemma 4 in the appendix. The proof follows the similar steps as before, simply replacing the bound for the $6 j$-symbols with the bound on the Barrett-Crane vertex amplitude (A24). More precisely, there are two kind of colorings corresponding to external and internal faces respectively. We denote by $N^{e}$ the colors labeling the ten external surfaces in the diagram of Fig. 3, while $N^{i}$ denotes the colors of the ten internal faces. Only internal colors are summed over. External colors label the surfaces shown on the left of Fig. 5. They appear in the propagator corresponding to the appropriate internal edge, thus contributing with a factor $\Delta_{N^{e}}^{-2}$ to the amplitude. They also appear as arguments of two vertex amplitudes. Using (A24) this contribution will be less or equal than $\Delta_{N^{e}}^{2}$. There will be no face contribution for external colors (the face contribution appears when one has a complete chain of propagators closing around a face so that all the $\delta_{\alpha \beta}$ in (52) combine into $\delta_{\alpha \alpha}=\Delta_{N}$ ). Therefore, external colors $N^{e}$ can appear in the bound for the amplitude only through the dimensionality of the space of intertwiners $\Delta_{N_{1}, \ldots, N_{4}}$ in each propagator associated to the ten internal edges. We denote the $\Delta_{N_{1}, \ldots, N_{4}}$, in (57), as $\Delta^{a b}$ for $a, b=1, \ldots, 5$ and $a \neq b$, according to Fig. 3 .

On the other hand, internal colors appear as face contributions $\left(\Delta_{N^{i}}\right)$. There is also a contribution from the three edges of each face $\left(\Delta_{N^{i}}^{-6}\right)$, and the contribution of the three corresponding vertices which are less or equal that $\Delta_{N^{i}}^{3}$, according to (A24).

From the considerations of the two previous paragraphs we obtain

$$
\left|A_{5}^{(b c)}\left(N_{1}^{e} \ldots N_{10}^{e}\right)\right| \leq \sum_{N_{1}^{i} \ldots N_{10}^{i}}\left(\Delta_{N_{1}^{i}} \ldots \Delta_{N_{10}^{i}}\right)^{-2} \prod_{a b} \Delta_{N^{e}, N^{i}}^{a b}
$$

In order to construct a manifestly finite upper bound for the amplitude we need to obtain a bound for $\Delta_{N_{1}, \ldots, N_{4}}$. To find an upper bound for $\Delta_{N_{1}, \ldots, N_{4}}$ we proceed as follows. The $\Lambda$ 's appear in (55) through $\phi_{N_{1} N_{2} N_{3} N_{4}, \Lambda_{1}}^{\alpha_{1} \ldots \alpha_{2}}$ where $\Lambda$ labels the elements of an orthonormal base of intertwiners between the four representations $N_{1} \ldots N_{4}$. Assume that $N_{1}$ is less or equal than the other $N_{i}$. We chose a basis in which for example $N_{1}$ and $N_{2}$ are in the same 3-intertwiner $C^{N_{1} N_{2} \Lambda}$ in (A3). Since $N_{1}, N_{2}$, and $\Lambda$ have to be $S O(4)$ compatible it follows that $N_{2}-N_{1} \leq \Lambda \leq N_{1}+N_{2}$. There are $2 N_{1}+1=\sqrt{\Delta_{N_{1}}} \Lambda$ 's who satisfy this condition. However, there are additional compatibility conditions on $\Lambda$, so we conclude that the number of possible $\Lambda$ 's denoted by $\Delta_{N_{1}, \ldots, N_{4}}$ satisfies $\Delta_{N_{1}, \ldots, N_{4}} \leq \sqrt{\Delta_{N_{1}}}$, since $N_{1}$ was the smallest $N_{i}$ we can also write

$$
\Delta_{N_{1}, \ldots, N_{4}} \leq \sqrt{\Delta_{N_{i}}}
$$

for $i=1,2,3,4$. The dimensionality of the space of intertwiners is independent of the chosen basis and the previous inequality has a basis independent meaning. We can use the colors of the ten internal faces $N^{i}$ to bound the ten $\Delta^{a b}$ 
in the previous equation. Finally, according to (64) we obtain

$$
\left|A_{5}^{(b c)}\left(N_{1}^{e} \ldots N_{10}^{e}\right)\right| \leq \sum_{N_{1}^{i} \ldots N_{10}^{i}}\left(\Delta_{N_{1}^{i}} \ldots \Delta_{N_{10}^{i}}\right)^{-\frac{3}{2}}<\infty .
$$

The same kind of inequality can be derived for $A_{4}^{b c}$.

\section{CONCLUSION}

We have presented in this paper a spin foam model, possibly related to Euclidean quantum gravity, in which the fundamental amplitudes, divergent in the BF and Barrett-Crane models, are finite. The model is obtained as a modification of the interaction term of quantum BF theory, formulated on the group manifold. The modification is an implementation, à la Barrett-Crane, of the constraint that reduced BF to general relativity.

We have not proven finiteness of all amplitudes at all orders in the expansion in 2-complexes, but we suspect that by using the methods introduced here the proof could be possible. For each particular amplitude, it is easy to construct a finite bound of the amplitude, using the inequalities given in the paper. The difficulty consists of finding a general way of bounding the degeneracy of the vertex amplitude given by the dimensionality of the space of intertwiners associated to the 5 edges converging at each vertex. In addition, the inequalities given in the appendix might be used to study the convergence of the full Feynman series, or sum over 2-complex.

It would be crucial to get a better understanding of the classical limit of the model. In particular, it would be interesting to know whether the amplitude of a spin foam approaches the exponential of the Einstein-Hilbert action of the four-geometry that the spin foam approximate.

More in general, we think that the techniques and methods introduced here could be useful for analyzing divergences in general spin foam models.

\section{ACKNOWLEDGMENTS}

A.P. thanks FUNDACION YPF (Argentina) for its support. This work was partially supported by NSF Grant PHY-9900791.

\section{APPENDIX A: IRREDUCIBLE REPRESENTATIONS OF $S O(4)$}

In this appendix we review some properties and definitions in the theory of irreducible representations of $\mathrm{SO}(4)$ (on this see [31]), and we state and prove some inequalities used in the paper to show the finiteness of amplitudes in previous sections. We follow the conventions of [23].

Given $g \in S O(4)$ we denote by $D_{\alpha \beta}^{(\Lambda)}(g)$ the representation matrix corresponding to the irreducible representation of order $\Lambda$. Integration over $S O(4)$ or the $S O(3)$ subgroup $H$ is performed with the normalized Haar measure of the group and the subgroup respectively. The integration of two representation matrices is given by

$$
\int_{S O(4)} d g \overline{D_{\alpha \beta}^{(\Lambda)}(g)} D_{\alpha^{\prime} \beta^{\prime}}^{\left(\Lambda^{\prime}\right)}(g)=\frac{1}{\Delta_{\Lambda}} \delta^{\Lambda \Lambda^{\prime}} \delta_{\alpha \alpha^{\prime}} \delta_{\beta \beta^{\prime}}
$$

where $\Delta(N)$ denotes the dimension of the representation. In the case of $S O(4)$ we can choose a basis in which matrices are orthogonal, and the bar can be dropped from the previous equation. The integral of the product of three group elements is

$$
\int_{S O(4)} d g D_{\alpha_{1} \beta_{1}}^{\left(N_{1}\right)}(g) D_{\alpha_{2} \beta_{2}}^{\left(N_{2}\right)}(g) D_{\alpha \beta}^{(\Lambda)}(g)=C_{\alpha_{1} \alpha_{2} \alpha}^{N_{1} N_{2} \Lambda} C_{\beta_{1} \beta_{2} \beta}^{N_{1} N_{2} \Lambda}
$$

Here $C_{\gamma_{1} \gamma_{2} \gamma}^{N_{1} N_{2} \Lambda}$ are normalized intertwiners (Wigner 3-j symbols) between three representations of $\mathrm{SO}(4)$; that is $C_{\alpha_{1} \alpha_{2} \alpha}^{N_{1} N_{2} \Lambda} C_{\alpha_{1} \alpha_{2} \alpha}^{N_{1} N_{2} \Lambda}=1$. The intertwiner from the tensor product of two representations $N_{1}, N_{2}$ to a representation $\Lambda$, if it exists is unique. 
The intertwiners between four representations are of great importance in our calculation. However, in general they are not unique and rather form a vector space. An orthonormal base can be defined as follows:

$$
C_{\gamma_{1} \ldots \gamma_{4}}^{N_{1} \ldots N_{4} \Lambda}=\sqrt{\operatorname{dim}_{\Lambda}} C_{\gamma_{1} \gamma_{2} \gamma}^{N_{1} N_{2} \Lambda} C_{\gamma_{3} \gamma_{4} \gamma}^{N_{3} N_{4} \Lambda}
$$

With these definitions equation (A2) generalizes to the case of the integration of four representation matrices to

$$
\int_{S O(4)} d g D_{\alpha_{1} \beta_{1}}^{\left(N_{1}\right)}(g) \ldots D_{\alpha_{4} \beta_{4}}^{\left(N_{4}\right)}(g)=\sum_{\Lambda} C_{\alpha_{1} \ldots \alpha_{4}}^{N_{1} \ldots N_{4} \Lambda} C_{\beta_{1} \ldots \beta_{4}}^{N_{1} \ldots N_{4} \Lambda} .
$$

Another important equation corresponds to the integration of one representation matrix over a sub-group $S O(3) \subset$ $S O(4)$, namely

$$
\int_{H=S O(3)} d h D_{\alpha \beta}^{(N)}(h)=w_{\alpha}^{(N)} w_{\beta}^{(N)}
$$

where $w_{\alpha}^{(N)}$ represents the unit vector in the irreducible representation of order $N$ left invariant by the action of the subgroup $H\left(w_{\alpha}^{(N)}\right.$ is non vanishing only if $N$ is simple). Equation (A5) defines the projector into that one-dimensional vector space.

As mentioned, invariant vectors exist only in simple representations. As a consequence the projection of the intertwiner $C_{\gamma_{1} \ldots \gamma_{4}}^{N_{1} \ldots N_{4}}{ } w_{\gamma_{1}} \ldots w_{\gamma_{4}}$ vanishes unless all the $N_{i}$ and $N$ are simple. In this case its value is given by

$$
C_{\gamma_{1} \ldots \gamma_{4}}^{N_{1} \ldots N_{4}} w_{\gamma_{1}} \ldots w_{\gamma_{4}}=\frac{1}{\sqrt{\Delta\left(N_{1}\right) \ldots \Delta\left(N_{4}\right)}}
$$

Finally we give the definition of the Barrett-Crane intertwiner:

$$
B_{\gamma_{1} \ldots \gamma_{4}}^{N_{1}, N_{2}, N_{3}, N_{4}} \equiv \sum_{N} C_{\gamma_{1} \ldots \gamma_{4}}^{N_{1} \ldots N_{4} N}
$$

The previous is the un-normalized Barrett-Crane intertwiner as originally defined in [7] which is shown to be unique up to scaling in [33, 32 .

\section{Some lemmas}

Now we state and prove the lemma that plays an important role in showing the finiteness of the 1-bubble amplitude in the modified Barrett-Crane model. We define the following distribution

$$
\mathcal{D}\left(g_{1}, g_{2}, g_{3}\right)=\int_{H^{3}} d h_{i} d \beta_{i} \delta\left(\beta_{1} h_{1} \beta_{1}^{-1} \beta_{2} h_{2} \beta_{2}^{-1} g_{1} \beta_{3} h_{3} \beta_{3}^{-1} \beta_{4} h_{4} \beta_{4}^{-1} g_{2} \beta_{5} h_{5} \beta_{5}^{-1} \beta_{6} h_{6} \beta_{6}^{-1} g_{3}\right)
$$

\section{Lemma 1:}

The distribution $\mathcal{D}\left(g_{1}, g_{2}, g_{3}\right)$ is a bounded function over $S O(4)^{3}$.

Proof: In order to prove the lemma we expand A $\mathrm{A}$ ) using

$$
\delta(g)=\sum_{N} \Delta(N) \operatorname{Tr}\left(D^{(N)}(g)\right)
$$

We integrate over $\beta_{i}$ and $h_{i}$ and make use of equations A5 and (A1). The mode expansion of $\mathcal{D}\left(g_{1}, g_{2}, g_{3}\right)$ results:

$$
\mathcal{D}\left(g_{1}, g_{2}, g_{3}\right)=\sum_{\text {Simple } \Lambda} \frac{1}{\Delta^{5}(\Lambda)} \operatorname{Tr}\left(D^{(\Lambda)}\left(g_{1} g_{2} g_{3}\right)\right)
$$

Now

$$
\left|\sum_{\text {Simple } \Lambda} \frac{1}{\Delta^{5}(\Lambda)} \operatorname{Tr}\left(D^{(\Lambda)}\left(g_{1} g_{2} g_{3}\right)\right)\right|<\sum_{\text {Simple } \Lambda} \frac{1}{\Delta^{5}(\Lambda)}\left|\operatorname{Tr}\left(D^{(\Lambda)}\left(g_{1} g_{2} g_{3}\right)\right)\right|<\sum_{\text {Simple } \Lambda} \frac{1}{\Delta^{4}(\Lambda)}<\infty
$$


where we have used that in our orthogonal representation $\left|\operatorname{Tr}\left(D^{\Lambda}(g)\right)\right| \leq \Delta_{\Lambda}$. Therefore, $\mathcal{D}\left(g_{1}, g_{2}, g_{3}\right)$ is bounded on $S O^{3}(4)$

By means of the previous lemma we were able to find a bound to the 1-bubble amplitude directly in configuration space. To show the finiteness of the 5-bubble amplitude in the previous section, we made use of the mode expansion of the amplitude and the following lemma?.

\section{Lemma 2:}

The $S O(4) 6 j$-symbols defined in terms of normalized intertwiners (normalized Wigner $3 j$-symbols $C_{\alpha_{1} \alpha_{2} \alpha_{3}}^{N_{1} N_{2} N_{3}}$ of (A2)) satisfy the following inequality:

$$
\left|\left\{\begin{array}{lll}
N_{1} & N_{2} & N_{3} \\
N_{4} & N_{5} & N_{6}
\end{array}\right\}_{6 j}\right| \leq \sqrt{\Delta_{N_{1}} \Delta_{N_{2}} \Delta_{N_{3}} \Delta_{N_{4}} \Delta_{N_{5}} \Delta_{N_{6}}}
$$

where $\Delta_{N}$ denotes the dimension of the irreducible representation of order $N$.

Proof: The $6 j$-symbol is defined in terms of $C_{\alpha_{1} \alpha_{2} \alpha_{3}}^{N_{1} N_{2} N_{3}}$ as

$$
\left\{\begin{array}{lll}
N_{1} & N_{2} & N_{3} \\
N_{4} & N_{5} & N_{6}
\end{array}\right\}_{6 j} \equiv C_{\alpha_{1} \alpha_{2} \alpha_{3}}^{N_{1} N_{2} N_{3}} C_{\alpha_{1} \alpha_{4} \alpha_{6}}^{N_{1} N_{4} N_{6}} C_{\alpha_{2} \alpha_{4} \alpha_{5}}^{N_{2} N_{4} N_{5}} C_{\alpha_{3} \alpha_{6} \alpha_{5}}^{N_{3} N_{6} N_{5}}
$$

where summation over repeated indices is understood. The assertion of the lemma is proven by means of calculating the following integral in two different ways.

$$
\begin{array}{r}
\mathcal{S}=\int d g_{1} d g_{2} d g_{3} d g_{4}\left(D\left(g_{1}\right)_{\alpha_{1} \beta_{1}}^{N_{1}} D\left(g_{1}\right)_{\alpha_{2} \beta_{2}}^{N_{2}} D\left(g_{1}\right)_{\alpha_{3} \beta_{3}}^{N_{3}}\right)\left(D\left(g_{2}\right)_{\alpha_{1} \beta_{1}}^{N_{1}} D\left(g_{2}\right)_{\alpha_{4} \beta_{4}}^{N_{4}} D\left(g_{2}\right)_{\alpha_{6} \beta_{6}}^{N_{6}}\right) \\
\left(D\left(g_{3}\right)_{\alpha_{2} \beta_{2}}^{N_{2}} D\left(g_{3}\right)_{\alpha_{4} \beta_{4}}^{N_{4}} D\left(g_{3}\right)_{\alpha_{5} \beta_{5}}^{N_{5}}\right)\left(D\left(g_{4}\right)_{\alpha_{3} \beta_{3}}^{N_{3}} D\left(g_{4}\right)_{\alpha_{6} \beta_{6}}^{N_{6}} D\left(g_{4}\right)_{\alpha_{5} \beta_{5}}^{N_{5}}\right)
\end{array}
$$

We can rewrite the previous equation using the representation property $D(g) D(f)=D(g f)$ as

$$
\begin{aligned}
\mathcal{S}=\int d g_{i} \operatorname{Tr}\left[D^{N_{1}}\left(g_{1} g_{2}^{-1}\right)\right] & \operatorname{Tr}\left[D^{N_{2}}\left(g_{1} g_{3}^{-1}\right)\right] \operatorname{Tr}\left[D^{N_{3}}\left(g_{1} g_{4}^{-1}\right)\right] \\
& \operatorname{Tr}\left[D^{N_{4}}\left(g_{2} g_{3}^{-1}\right)\right] \operatorname{Tr}\left[D^{N_{5}}\left(g_{3} g_{4}^{-1}\right)\right] \operatorname{Tr}\left[D^{N_{6}}\left(g_{2} g_{4}^{-1}\right)\right] .
\end{aligned}
$$

The fact that we are using orthogonal irreducible representations of $S O(4)$ implies that $\left|\operatorname{Tr}\left[D^{N}(g)\right]\right| \leq \Delta_{N}$. Combining this bound for the trace with the normalization of the $S O(4)$ Haar measure in the previous equation we conclude that

$$
|\mathcal{S}| \leq \Delta_{N_{1}} \Delta_{N_{2}} \Delta_{N_{3}} \Delta_{N_{4}} \Delta_{N_{5}} \Delta_{N_{6}}
$$

On the other hand, using equation (A2), and the definition of the $6 j$-symbol above we obtain

$$
\mathcal{S}=\left\{\begin{array}{lll}
N_{1} & N_{2} & N_{3} \\
N_{4} & N_{5} & N_{6}
\end{array}\right\}_{6 j}^{2}
$$

which concludes the proof $\square$

\section{Lemma 3:}

The $S 0(4) 15 j$-symbols defined in terms of normalized intertwiners $\left(C_{\alpha_{1} \alpha_{2} \alpha_{3} \alpha_{4}}^{N_{1} N_{2} N_{3} N_{4}, \Lambda}\right.$ of (A4)) satisfy the following inequality:

$$
\sum_{\Lambda_{1} \ldots \Lambda_{10}}\left\{\begin{array}{ccccc}
N_{1} & N_{2} & N_{3} & N_{4} & N_{5} \\
N_{6} & N_{7} & N_{8} & N_{9} & N_{10} \\
\Lambda_{1} & \Lambda_{2} & \Lambda_{3} & \Lambda_{4} & \Lambda_{5}
\end{array}\right\}_{15 j}^{2} \leq \Delta_{N_{1}} \Delta_{N_{2}} \Delta_{N_{3}} \Delta_{N_{4}} \Delta_{N_{5}} \Delta_{N_{6}} \Delta_{N_{7}} \Delta_{N_{8}} \Delta_{N_{9}} \Delta_{N_{10}}
$$

where

\footnotetext{
${ }^{2}$ We have not found a reference to this lemma in the literature, although we suspect is must be a known result due to its simplicity.
} 


$$
\left\{\begin{array}{ccccc}
N_{1} & N_{2} & N_{3} & N_{4} & N_{5} \\
N_{6} & N_{7} & N_{8} & N_{9} & N_{10} \\
\Lambda_{1} & \Lambda_{2} & \Lambda_{3} & \Lambda_{4} & \Lambda_{5}
\end{array}\right\}_{15 j}:=C_{\alpha_{1} \alpha_{2} \alpha_{3} \alpha_{4}}^{N_{1} N_{2} N_{3} N_{4}, \Lambda_{1}} C_{\alpha_{4} \alpha_{5} \alpha_{6} \alpha_{7}}^{N_{4} N_{5} N_{6} N_{7}, \Lambda_{2}} C_{\alpha_{7} \alpha_{3} \alpha_{8} \alpha_{9}}^{N_{7} N_{3} N_{8} N_{9}, \Lambda_{3}} C_{\alpha_{9} \alpha_{6} \alpha_{2} \alpha_{10}}^{N_{9} N_{6} N_{2} N_{10}, \Lambda_{4}} C_{\alpha_{10} \alpha_{8} \alpha_{5} \alpha_{1}}^{N_{10} N_{8} N_{5} N_{1}, \Lambda_{5}}
$$

This lemma generalizes the previous one; however for simplicity we have proven explicitly the first. The proof of the current lemma follows the analogous path as the previous one with some additional indices. One starts by defining an integral analogous to $\mathcal{S}$ in lemma(2); namely,

$$
\begin{aligned}
\mathcal{S}= & \int d g_{1} d g_{2} d g_{3} d g_{4} d g_{5} D\left(g_{1}\right)_{\alpha_{1} \beta_{1}}^{N_{1}} D\left(g_{1}\right)_{\alpha_{2} \beta_{2}}^{N_{2}} D\left(g_{1}\right)_{\alpha_{3} \beta_{3}}^{N_{3}} D\left(g_{1}\right)_{\alpha_{4} \beta_{4}}^{N_{4}} D\left(g_{2}\right)_{\alpha_{4} \beta_{4}}^{N_{4}} D\left(g_{2}\right)_{\alpha_{5} \beta_{5}}^{N_{5}} \\
& D\left(g_{2}\right)_{\alpha_{6} \beta_{6}}^{N_{6}} D\left(g_{2}\right)_{\alpha_{7} \beta_{7}}^{N_{7}} D\left(g_{3}\right)_{\alpha_{7} \beta_{7}}^{N_{7}} D\left(g_{3}\right)_{\alpha_{3} \beta_{3}}^{N_{3}} D\left(g_{3}\right)_{\alpha_{8} \beta_{8}}^{N_{8}} D\left(g_{3}\right)_{\alpha_{9} \beta_{9}}^{N_{9}} D\left(g_{4}\right)_{\alpha_{9} \beta_{9}}^{N_{9}} D\left(g_{4}\right)_{\alpha_{6} \beta_{6}}^{N_{6}} \\
& D\left(g_{4}\right)_{\alpha_{2} \beta_{2}}^{N_{2}} D\left(g_{4}\right)_{\alpha_{10} \beta_{10}}^{N_{10}} D\left(g_{5}\right)_{\alpha_{10} \beta_{10}}^{N_{10}} D\left(g_{5}\right)_{\alpha_{8} \beta_{8}}^{N_{8}} D\left(g_{5}\right)_{\alpha_{5} \beta_{5}}^{N_{5}} D\left(g_{5}\right)_{\alpha_{1} \beta_{1}}^{N_{1}} \cdot
\end{aligned}
$$

where now one has an integration over five group variables of the appropriate product of twenty representation matrices. One can relate the value of the integral to the sum of $15 j$-symbols squared over the $\Lambda$ 's by means of (A4). Finally, one finds a bound to the integral using the fundamental inequality $\left|\operatorname{Tr}\left[D^{N}(g)\right]\right| \leq \Delta_{N}$.

Now we state two corollaries of the previous lemma. First, we have that

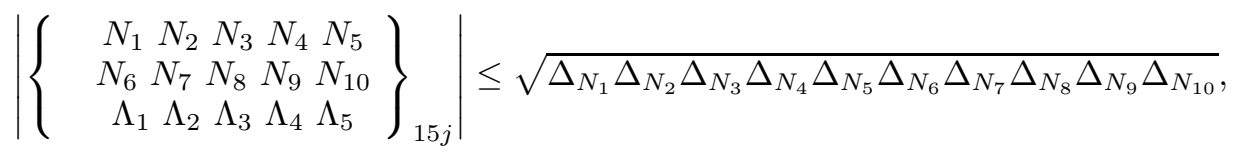

and second, from the definition of the Barrett-Crane vertex we conclude that

$$
\left|\mathcal{B}_{N_{1}, \ldots, N_{10}}\right| \leq\left(\sum_{\Lambda_{1} \ldots \Lambda_{5}} 1\right) \sqrt{\Delta_{N_{1}} \Delta_{N_{2}} \Delta_{N_{3}} \Delta_{N_{4}} \Delta_{N_{5}} \Delta_{N_{6}} \Delta_{N_{7}} \Delta_{N_{8}} \Delta_{N_{9}} \Delta_{N_{10}}}
$$

An important consequence of $(\mathrm{A} 20)$ is that it can also be used to directly find a bound for the Barrett-Crane vertex. Take the integral defined in (A20) in which the $\beta_{i}$ are not contracted between them self as in (A20) but rather contracted to the appropriate set of normalized invariant vectors $w^{\beta_{i}}$ in each representation. Using (A4) and (A6) we obtain, on the one hand

$$
\frac{\mathcal{B}_{N_{1}, \ldots, N_{10}}}{\left(\Delta_{N_{1}} \Delta_{N_{2}} \Delta_{N_{3}} \Delta_{N_{4}} \Delta_{N_{5}} \Delta_{N_{6}} \Delta_{N_{7}} \Delta_{N_{8}} \Delta_{N_{9}} \Delta_{N_{10}}\right)}
$$

However, on the other hand, the absolute value of integrand contracted with all the w's is less or equal than one since it can be written as a product of terms of the form $\left|w^{\mu} D(g)_{\mu \nu} w^{\mu}\right| \leq 1$. Therefore, we have proven the following lemma:

\section{Lemma 4:}

The Barrett-Crane vertex amplitude satisfies the following inequality:

$$
\left|\mathcal{B}_{N_{1}, \ldots, N_{10}}\right| \leq \Delta_{N_{1}} \Delta_{N_{2}} \Delta_{N_{3}} \Delta_{N_{4}} \Delta_{N_{5}} \Delta_{N_{6}} \Delta_{N_{7}} \Delta_{N_{8}} \Delta_{N_{9}} \Delta_{N_{10}}
$$

[1] For an overview of the present approaches to the problem of the description of quantum spacetime, see: C Rovelli, Strings, loops and the others: a critical survey on the present approaches to quantum gravity in Gravitation and Relativity: At the turn of the Millennium, N Dadhich J Narlikar eds, pg 281-331 (Inter-University centre for Astronomy and Astrophysics, Pune 1998); gr-qc/9803024. For a discussion on quantum gravity and background independence, see: C Rovelli, The century of the incomplete revolution: searching for a general relativistic quantum field theory, J Math Phys (Special Issue 2000) 41 (2000) 3776, hep-th/9910131.

[2] M Reisenberger, Worldsheet formulations of gauge theories and gravity, talk given at the 7th Marcel Grossmann Meeting Stanford, July 1994; gr-qc/9412035. 
[3] J Iwasaki, A definition of the Ponzano-Regge quantum gravity model in terms of surfaces, J Math Phys 36 (1995) 6288.

[4] J Baez, Spin Foam Models, Class Quant Grav 15 (1998) 1827-1858; gr-qc/9709052. An Introduction to Spin Foam Models of Quantum Gravity and BF Theory, to appear in to appear in Geometry and Quantum Physics, eds Helmut Gausterer and Harald Grosse, Lecture Notes in Physics (Springer-Verlag, Berlin); gr-qc/9905087.

[5] M Reisenberger, C Rovelli, Sum over Surfaces form of Loop Quantum Gravity, Phys Rev D56 (1997) 3490-3508. C Rovelli, Quantum gravity as a sum over surfaces, Nucl Phys B57 (1997) 28-43. C Rovelli, The projector on physical states in loop quantum gravity, gr-qc/9806121.

[6] R De Pietri, Canonical em Loop Quantum Gravity and Spin Foam Models, Proceeding of the XXIII SIGRAV conference, Monopoli (Italy), September 1998.

[7] JW Barrett and L Crane, Relativistic spin networks and quantum gravity, J Math Phys 39 (1998) 3296.

[8] C Misner, Feynman quantization of General relativity, Rev Mod Phys 29 (1957) 497.

[9] SW Hawking, em The Path-Integral Approach to Quantum Gravity, in General Relativity: An Einstein Centenary Survey, SW Hawking and W Israel eds. (Cambridge University Press, Cambridge 1979).

[10] G Ponzano, T Regge, in Spectroscopy and Group Theoretical Methds in Physics, F Block Ed, (North Holland, New York 1968) pp 1-58; On the relation with canonical quantum gravity, see: C Rovelli, Basis of the Ponzano-Regge-Turaev-ViroOoguri quantum gravity model is the loop representation basis, Phys Rev D48 (1993) 2702. TJ Foxon, Spin networks, Turaev-Viro theory and the loop representation, Class Quant Grav 12 (1995) 951-964, gr-qc/9408013.

[11] C Rovelli, L Smolin, Knot theory and quantum gravity, Phys Rev Lett 61 (1988) 1155. C Rovelli, L Smolin, Loop space representation of quantum general relativity, Nucl Phys, B331 (1990) 80. For a review, see: C Rovelli, Loop Quantum Gravity, Living Reviews in Relativity (refereed electronic journal), invited review paper. http://www.livingreviews.org Articles/Volume1/1998-1rovelli; gr-qc/9709008. For an introduction, see: M Gaul, C Rovelli, Loop quantum gravity and the meaning of diffeomorphism invariance, in Towards Quantum Gravity. Proceedings of the XXXV Karpacz International Winter School on Theoretical Physics J Kowalski-Glikman ed (Springer Verlag, Heidelberg, 1999).

[12] C Rovelli, L Smolin, Spin Networks and Quantum Gravity, Phys Rev D53 (1995) 5743-5759. C Rovelli and L Smolin, Discreteness of Area and Volume in Quantum Gravity, Nucl Phys B442 (1995) 593, gr-qc/9411005; Erratum: Nucl Phys B456 (1995) 734. JC Baez, Adv in Math Phys 117 (1996) 253; gr-qc/9411007. JC Baez, in The Interface of Knots and Physics, LH Kauffman ed, (American Mathematical Society, Providence, Rhode Island, 1996), gr-qc/9504036.

[13] F Markopoulou, L Smolin, Causal evolution of spin networks, Nucl Phys B508 (1997) 409-430.

[14] M Reisenberger, A left-handed simplicial action for Euclidean general relativity, Class and Quantum Grav 14 (1997) 1730-1770; gr-qc/9609002; gr-qc/9711052; gr-qc/9903112

[15] L Freidel, K Krasnov Spin Foam Models and the Classical Action Principle, Adv Theor Math Phys 2 (1999) $1183-1247$.

[16] J Iwasaki A surface theoretic model of quantum gravity, gr-qc/9903112. J Iwasaki,A lattice quantum gravity model with surface-like excitations in 4-dimensional spacetime, gr-qc/0006088.

[17] V Turaev, Quantum invariants of 3-manifolds and a glimpse of shadow topology in Quantum Groups, Springer Lecture Notes in Mathematics 1510, pp 363-366 (Springer-Verlag, New York, 1992); Quantum Invariants of Knots and 3-Manifolds (de Gruyter, New York, 1994).

[18] H Ooguri, Topological Lattice Models in Four Dimensions, Mod Phys Lett A7 (1992) 2799.

[19] L Crane and D Yetter, A Categorical construction of 4-D topological quantum field theories, in Quantum Topology, L Kaufmann and R Baadhio eds, World Scientific, Singapore 1993; hep-th/9301062.

[20] L Crane, L Kauffman and D Yetter, J Knot Theor Ramifications 6 (1997) 177-234; hep-th/9409167.

[21] R Reisenberger gr-qc/9412035, gr-qc/9609002. J Baez, em 4-Dimensional BF Theory as a Topological Quantum Field Theory, Lett Math Phys 38 (1996) 128. H Waelbroeck, JA Zapata gr-qc/9211035

[22] L Crane, Topological field theory as the key to quantum gravity, Proceedings of the conference on knot theory and quantum gravity, Riverside, J Baez ed 1992. J Barret Quantum Gravity as Topological Quantum Field Theory J Math Phys 36 (1995) 6161-6179. L Smolin, Linking Topological Quantum Field Theory and Nonperturbative Quantum Gravity, J Math Phys 36 (1995) 6417.

[23] R De Pietri, L Freidel, K Krasnov, C Rovelli, Barrett-Crane model from a Boulatov-Ooguri field theory over a homogeneous space, Nuclear Physics, to appear, hep-th/9907154.

[24] M Reisenberger, C Rovelli, Spinfoam models as Feynman diagrams, gr-qc/0002083. M Reisenberger, C Rovelli, Spacetime as a Feynman diagram: the connection formulation, gr-qc/0002095.

[25] R De Pietri, C Petronio, Feynman Diagrams of Generalized Matrix Models and the Associated Manifolds in Dimension 4, gr-qc/0004045.

[26] E Brézin, C Itzykson, G Parisi, J Zuber, Commun Math Phys 59 (1978) 35. F David, Nucl Phys B257 (1985) 45. J Ambjorn, B Durhuus, J Frolich, Nucl Phys B257 (1985) 433. VA Kazakov, IK Kostov, AA Migdal, Phys Lett 157 (1985) 295. D V Boulatov, V A Kazakov, I K Kostov, A A Migdal, Nucl Phys B275 (1986) 641. M Douglas, S Shenker, Nucl Phys B335(1990) 635. D Gross, A Migdal, Phys Rev Lett 64 (1990) 635; E Brezin, V A Kazakov, Phys Lett B236 (1990) 144. For the passage from 2d models to field theories on a group, see DV Boulatov, Mod Phys Lett A7 (1992) 1629.

[27] A Perez, C Rovelli, Bubbles and renormalization of spin foams, in preparation.

[28] V Turaev, O Viro, State sum invariant of 3-manifolds and quantum 6j-symbols Topology 31 (1992) 865-902.

[29] JC Baez, JW Barrett, The Quantum Tetrahedron in 3 and 4 Dimensions, gr-qc/9903060. 
[30] A Barbieri, Quantum tetrahedra and simplicial spin networks, Nucl Phys B518 (1998) 714.

[31] NJ Vilenkin and AU Klimyk, Representation of Lie Groups and Special Functions (Kluwer Academic Publisher, Dordrecht, The Netherland, 1993), Vol 2, volume 2: Class I Representations, Special Functions, and Integral Transforms.

[32] A Barbieri, Space of the vertices of relativistic spin networks, gr-qc/9709076.

[33] MP Reisenberger, On relativistic spin network vertices, J Math Phys 40 (1999) 2046-2054.

[34] F Markopoulou, An algebraic approach to coarse graining, hep-th/0006199. 